\title{
On the Weak Admissibility of Singular Perturbations in Cauchy Problems
}

\author{
By \\ Ryuichi AsHINo*
}

\section{Introduction}

In [3], we have studied conditions on operators for the convergence of solutions of singulary perturbed non-characteristic Cauchy problems. The convergence of solutions depends on the Cauchy data. In this paper, we shall mainly study necessary and sufficient conditions on the Cauchy data for the convergence of solutions of a given one-parameter family of singulary perturbed Cauchy problems.

Let $P_{1}(D)$ and $P_{2}(D)$ be linear differential operators with constant coefficients. Let the order of $P_{1}$ be $m$ and that of $P_{2}$ be $m^{\prime}$. Assume that $m>m^{\prime}$. For $x=\left(x_{1}, \cdots, x_{n}\right)$ in $\boldsymbol{R}^{n}$, put $x^{\prime}=\left(x_{2}, \cdots, x_{n}\right)$. Let us consider the following one-parameter family of Cauchy problems:

$$
\left\{\begin{array}{l}
\left(\varepsilon^{m-m^{\prime}} \cdot P_{1}(D)+P_{2}(D)\right) u(x)=0, \quad \text { in } \quad \boldsymbol{R}^{n} ; \\
\left.D_{1}^{j-1} u(x)\right|_{x_{1}=0}=\phi_{j}\left(x^{\prime}\right), \quad j=1, \cdots, m,
\end{array}\right.
$$

where $\varepsilon$ is a small positive parameter. In [2], we have studied that if the solutions $u_{\mathrm{g}}$ of (1.1) converge in a suitable topology, then the limit satisfies the following reduced problem:

$$
\begin{cases}P_{2}(D) u=0, & \text { in } \quad \boldsymbol{R}^{n} ; \\ \left.D_{1}^{j-1} u\right|_{x_{1}=0}=\phi_{j}, & j=1, \cdots, m^{\prime} .\end{cases}
$$

Let the symbols of $P_{1}(D)$ and $P_{2}(D)$ be

$$
\begin{aligned}
& P_{1}(\xi)=\sum_{j=0}^{m} p_{1, j}\left(\xi^{\prime}\right) \cdot \xi_{1}^{m-j}, \\
& P_{2}(\xi)=\sum_{j=0}^{m^{\prime}} p_{2, j}\left(\xi^{\prime}\right) \cdot \xi_{1}^{m^{\prime}-j} .
\end{aligned}
$$

Communicated by S. Matsuura, January 27, 1989. Revised April 11, 1989.

* Research Institute for Mathematical Sciences Kyoto University, Kyoto, Japan. 
Assume that $p_{1,0}$ is a non-zero constant and ord $p_{1, j}\left(\xi^{\prime}\right) \leqq j, j=1, \cdots, m$ and that $p_{2,0}$ is a non-zero constant and ord $p_{2, j}\left(\xi^{\prime}\right) \leqq j, j=1, \cdots, m^{\prime}$. Such an operator satisfying the above conditions is said to be kowalewskian. Denote the characteristic roots of $\varepsilon^{m-m^{\prime}} \cdot P_{1}(\xi)+P_{2}(\xi)=0$ with respect to $\xi_{1}$ by $\tau_{j}\left(\varepsilon, \xi^{\prime}\right), j=1, \cdots$, $m$, those of $P_{1}(\xi)=0$ by $v_{j}\left(\xi^{\prime}\right), j=1, \cdots, m$, and those of $P_{2}(\xi)=0$ by $\sigma_{j}\left(\xi^{\prime}\right)$, $j=1, \cdots, m^{\prime}$.

Assumption 1.1. There exists a point $\xi_{0}^{\prime}$ in $\mathbb{R}^{n-1}$ such that for any $j, k$, $1 \leqq j<k \leqq m^{\prime}$

$$
\sigma_{j}\left(\xi_{0}^{\prime}\right) \neq \sigma_{k}\left(\xi_{0}^{\prime}\right)
$$

Remark. If Assumption 1.1 is satisfied, then there exists an open ball $B_{0}=B\left(r_{0} ; \xi_{0}^{\prime}\right)$ of radius $r_{0}$ with the centre $\xi_{0}^{\prime}$ such that all $\sigma_{j}\left(\xi^{\prime}\right)$ are distinct on the closure of $B_{0}$.

Condition 1.2. $\left(m-m^{\prime}=2\right.$ and $\left.p_{2,0} / p_{1,0}<0\right)$ or $\left(m-m^{\prime}=1\right.$ and $p_{2,0} / p_{1,0}$ is real)。

Remark. In [3], we have studied that the characteristic roots $\tau_{j}\left(\varepsilon, \xi^{\prime}\right)$ are classified into two classes as follows. One consists of all $\tau_{j}\left(\varepsilon, \xi^{\prime}\right)$ which converge uniformly on every compact subset of $\boldsymbol{R}_{\xi^{\prime}}^{n-1}$ to one of $\sigma_{j}\left(\xi^{\prime}\right), j=1, \cdots, m^{\prime}$ when $\varepsilon \downarrow 0$ and the other consists of all $\tau_{j}\left(\varepsilon, \xi^{\prime}\right)$ which diverge for every fixed $\xi^{\prime}$ when $\varepsilon \downarrow 0$. Condition 1.2 is equivalent to the condition that the leading terms with respect to $\varepsilon$ of all the diverging characteristic roots are real.

Denote $\Phi^{\prime}=\left(\phi_{1}, \cdots, \phi_{m^{\prime}}\right), \Phi^{\prime \prime}=\left(\phi_{m^{\prime}+1}, \cdots, \phi_{m}\right)$, and $\Phi=\left(\Phi^{\prime}, \Phi^{\prime \prime}\right)$. Let the Cauchy problems (1.1) be given and the Cauchy data space $\mathcal{A}$ consisting of $\Phi$ be so chosen that for every sufficiently small $\varepsilon$ the Cauchy problem (1.1) with $\mathcal{A}$ is uniquely solvable. If the solutions $u_{\varepsilon}$ of the Cauchy problems (1.1) for $\Phi$ in $\mathcal{A}$ converge in a suitable topology when $\varepsilon \downarrow 0$, then we may say that the one-parameter family (1.1) is a singular perturbation. This is the reason why we introduced the notion of "admissibility" of singular perturbations in Cauchy problems in [3]. Let the Cauchy problem (1.2) be given and the Cauchy data space $\mathcal{A}^{\prime}$ consisting of $\Phi^{\prime}$ be so chosen that the Cauchy problem (1.2) with $\mathscr{A}^{\prime}$ is uniquely solvable. We choose the extra Cauchy data space $\mathcal{A}^{\prime \prime}$ consisting of $\Phi^{\prime \prime}$ so that for every sufficiently small $\varepsilon$ the Cauchy problem (1.1) with $\mathcal{A}=\mathcal{A}^{\prime} \times \mathcal{A}^{\prime \prime}$ can be solved uniquely. If for every $\Phi^{\prime}$ in $\mathscr{A}^{\prime}$ there exists $\Phi^{\prime \prime}$ in $\mathscr{A}^{\prime \prime}$ such that the solutions $u_{\mathrm{g}}$ of the Cauchy problems (1.1) for $\Phi=\left(\Phi^{\prime}, \Phi^{\prime \prime}\right)$ converge when $\varepsilon \downarrow 0$, then the solution of the 
Cauchy problem (1.2) for $\Phi^{\prime}$ in $\mathcal{A}^{\prime}$ can be represented as the limit of the solutions $u_{\mathrm{z}}$ of the Cauchy problems (1.1) for a datum $\Phi$ in $\mathcal{A}$. This is the reason why we shall introduce the notion of "weak admissibility" of singular perturbations in Cauchy problems.

Let $U^{\prime}$ be a domain of $\boldsymbol{R}_{x^{\prime}}^{n-1}$ and $\delta$ be a positive number.

Definition 1.3. The uniquely solvable Cauchy problems $(1.1)$ in $(-\delta, \delta) \times U^{\prime}$ with the Cauchy data space $\mathcal{A}$ are said to be $C$-admissible in $(-\delta, \delta) \times U^{\prime}$ as a singular perturbation with respect to a given uniquely solvable Cauchy problem (1.2) if for every Cauchy datum $\Phi$ in $\mathcal{A}$, the solutions of (1.1) converge to that of $(1.2)$ in $C\left((-\delta, \delta) \times U^{\prime}\right)$ and said to be $C$-weakly admissible in $(-\delta, \delta) \times U^{\prime}$ if for every Cauchy datum $\Phi^{\prime}$ in $\mathcal{A}^{\prime}$ there exists a datum $\Phi^{\prime \prime}$ in $\mathcal{A}^{\prime \prime}$ such that the solutions of (1.1) converge to that of $(1.2)$ in $C\left((-\delta, \delta) \times U^{\prime}\right)$.

Remark. Relacing $C\left((-\delta, \delta) \times U^{\prime}\right)$ by $L^{2}\left((-\delta, \delta) \times U^{\prime}\right)$, we can also define the $L^{2}$-admissibility and the $L^{2}$-weak admissibility.

Denote by $\mathcal{O}\left(\boldsymbol{C}^{n-1}\right)$ the space of entire functions defined in $\boldsymbol{C}^{n-1}$ and by $A\left(\boldsymbol{R}^{n-1}\right)$ the space of real analytic functions defined in $\boldsymbol{R}^{n-1}$. When $\mathcal{A}=$ $\mathcal{O}\left(C^{n-1}\right)^{m}$, the Cauchy-Kowalewski theorem assures the analytic unique solvability in $\boldsymbol{R}^{n}$ of (1.1) for sufficiently small $\varepsilon$. But even when $\mathcal{A}=A\left(\boldsymbol{R}^{n-1}\right)^{m}$, we can not prove that there exists a region independent of $\varepsilon$ such that the analytic solutions of (1.1) exist in the region. Hence we must choose $\mathcal{A}$ so as to be included in $\mathcal{O}\left(\boldsymbol{C}^{n-1}\right)^{m}$. On the other hand, we shall use the Fourier transformation. Therefore, we shall only study the $C$-weak admissibility in $\boldsymbol{R}^{n}$ when $\mathcal{A}=\mathrm{F}^{-1}\left(C_{0}^{\infty}\left(B_{0}\right)\right)^{m}$, where $\mathrm{F}^{-1}$ denotes the inverse Fourier transformation and $B_{0}$ is the open ball in Remark to Assumption 1.1.

The main result in [3] is that Condition 1.2 is necessary and sufficient for the $C$-admissibility of the Cauchy problems (1.1) in $\boldsymbol{R}^{n}$ with $\mathrm{F}^{-1}\left(C_{0}^{\infty}\left(B_{0}\right)\right)^{m}$. The main result in this paper is the following:

Main Theorem. Assume that Condition 1.2 is not satisfied. Let Assumption 1.1 be satisfied and $B_{0}$ be the open ball in Remark to Assumption 1.1. Denote $m^{\prime \prime}=m-m^{\prime}$. Let $\mathcal{A}^{\prime}=\mathrm{F}^{-1}\left(C_{0}^{\infty}\left(B_{0}\right)\right)^{m^{\prime}}$ and $\mathcal{A}^{\prime \prime}=\mathrm{F}^{-1}\left(C_{0}^{\infty}\left(B_{0}\right)\right)^{m^{\prime \prime}}$. Then the Cauchy problems (1.1) in $\boldsymbol{R}^{n}$ with $\mathcal{A}$ are $C$-weakly admissible in $\boldsymbol{R}^{n}$ if and only if $P_{1}(\xi)$ is divided by $P_{2}(\xi)$ in the polynomial ring $C[\xi]$. In this case, the solutions $u_{\mathrm{g}}(x ; \Phi)$ of $(1.1)$ are identically equal to the solution $u_{0}\left(x ; \Phi^{\prime}\right)$ of $(1.2)$.

In this paper, we shall always assume that Condition 1.2 is not satisfied. The proof of Main Theorem can be found in $\S 5$. 
When the Cauchy data space $\mathcal{A}$ is $\{(0, \cdots, 0)\}$, all the Cauchy problems (1.1) are $C$-admissible. Our another purpose is to seek the Cauchy data spaces $\mathcal{A}$ included in $\mathrm{F}^{-1}\left(C_{0}^{\infty}\left(B_{0}\right)\right)^{m}$ with which the Cauchy problems (1.1) are $C$-admissible. The results are stated in Theorem A and B in $\S 4$. Theorem $A$ will be used in the proof of Main Theorem.

In $\S 2$, we shall list up notation and study algebraic lemmas. In $\S 3$, we shall study a necessary and sufficient condition on the Cauchy data for the convergence of the solutions, but this condition depends on $\varepsilon$. We shall show that the convergence of the solutions implies that $\Phi^{\prime \prime}$ is uniquely determined by $\Phi^{\prime}$. In $\S 4$, we shall remove the dependency on $\varepsilon$ from this condition and distinguish the Cauchy data with which the solutions converge in the following two special cases.

(1) Theorem A. The case when $m^{\prime}$ or $m^{\prime}-1$ characteristic roots of $F_{2}(\xi)=0$ are equal to the characteristic roots of $P_{1}(\xi)=0$ in $B_{0}$.

(2) Theorem B. The case when $m^{\prime}=1$ or $\left(m^{\prime}=2\right.$ and $\left.m \geqq 4\right)$.

Roughly speaking, when $P_{1}(\xi)$ is divided by $P_{2}(\xi)$ in the polynomial ring $\mathbb{C}[\xi], \Phi^{\prime}$ can be free but otherwise $\Phi^{\prime}$ is restricted.

The same results as for $\mathcal{A}=\mathrm{F}^{-1}\left(C_{0}^{\infty}\left(B_{0}\right)\right)^{m}$ remain true for $\mathcal{A}=\mathrm{F}^{-1}\left(C_{0}\left(B^{0}\right)\right)^{m}$ but fail for $\mathscr{A}=\mathrm{F}^{-1}\left(\mathcal{E}^{\prime}\left(B_{0}\right)\right)^{m}$ in general.

\section{Preliminaries}

In this section, we shall state algebraic lemmas which will be needed. Similar calculation can be found in computation of the characters of the classical groups. See Chapter VII, [4].

Notation 2.1. Let $X_{j}, j=1, \cdots, m$ be variables and $S_{k}\left(X_{1}, \cdots, X_{m}\right)$, $k=1, \cdots, m$ be the elementary symmetric polynomials of $X_{j} j=1, \cdots, m$, that is, for $k=1, \cdots, m$

$$
S_{k}\left(X_{1}, \cdots, X_{m}\right)=\sum_{i_{1}<i_{2}<\cdots<i_{k}} X_{i_{1}} X_{i_{2}} \cdots X_{i_{k}}
$$

Put

$$
S_{0}\left(X_{1}, \cdots, X_{m}\right)=1
$$

and for $k \leqq-1$ or $k \geqq m+1$

$$
S_{k}\left(X_{1}, \cdots, X_{m}\right)=0 .
$$

Denote 


$$
D_{0}\left(X_{1}, \cdots, X_{m}\right)=\left|\begin{array}{ccc}
X_{1}^{0} \cdots \cdots & X_{m}{ }^{0} \\
\vdots & & \vdots \\
\dot{X}_{1}{ }^{m-1} & \cdots & X_{m}{ }^{m-1}
\end{array}\right|,
$$

for $k=1, \cdots, m$ and for functions $\phi_{j}, j=1, \cdots, m$

$$
\begin{aligned}
& D_{k}=D_{k}\left(X_{1}, \cdots, X_{m} ; \phi_{1}, \cdots, \phi_{m}\right) \\
& =\left|\begin{array}{ccccccc}
X_{1}^{0} & \cdots \cdots & X_{k-1}{ }^{0} & \phi_{1} & X_{k+1}{ }^{0} & \cdots \cdots & X_{m}{ }^{0} \\
\vdots & & \vdots & \vdots & \vdots & \\
X_{1}^{m-1} & \cdots & X_{k-1}{ }^{m-1} & \phi_{m} & X_{k+1}{ }^{m-1} & \cdots & X_{m}{ }^{m-1}
\end{array}\right|,
\end{aligned}
$$

and for $k=0, \cdots, m-1$

$$
D^{m-1-k}=D^{m-1-k}\left(X_{1}, \cdots, X_{m-1}\right)=\left|\begin{array}{cccc}
X_{1}{ }^{0} & \cdots & X_{m-1}{ }^{0} \\
\vdots & & \vdots \\
X_{1}^{k-1} & \cdots & X_{m-1}{ }^{k-1} \\
& & \\
X_{1}^{k+1} & \cdots & X_{m-1}{ }^{k+1} \\
\vdots & & \vdots \\
X_{1}{ }^{m-1} & \cdots & X_{m-1}{ }^{m-1}
\end{array}\right| .
$$

Denote $\zeta=\exp \frac{2 \pi i}{m-m^{\prime}}$ and $\tau_{j}^{\prime}=\zeta^{j-m^{\prime}-1}, j=m^{\prime}+1, \cdots, m$. Denote by $\theta$ the argument of $-p_{2,0} / p_{1,0}$ satisfying $0 \leqq \theta<2 \pi$ and put $\Theta=\exp \frac{i \theta}{m-m^{\prime}}$.

Lemma 2.2. Assume that $X_{i} \neq X_{j} 1 \leqq i<j \leqq m$. Then, for $j=1, \cdots, m-1$

$$
D^{j}\left(X_{1}, \cdots, X_{m-1}\right) / D^{0}\left(X_{1}, \cdots, X_{m-1}\right)=S_{j}\left(X_{1}, \cdots, X_{m-1}\right),
$$

and for $k=1, \cdots, m$

$$
\begin{aligned}
& D_{k}\left(X_{1}, \cdots, X_{m} ; \phi_{1}, \cdots, \phi_{m}\right) / D_{0}\left(X_{1}, \cdots, X_{m}\right) \\
& \quad=\frac{\sum_{j=0}^{m-1}(-1)^{j} \cdot S_{j}\left(X_{1}, \cdots, X_{k-1}, X_{k+1}, \cdots, X_{m}\right) \cdot \phi_{m-j}}{\sum_{j=0}^{m-1}(-1)^{j} \cdot S_{j}\left(X_{1}, \cdots, X_{k-1}, X_{k+1}, \cdots, X_{m}\right) \cdot X_{k}^{m-1-j}} .
\end{aligned}
$$

Proof. Expand the Vandermonde determinant $D_{0}\left(X_{1}, \cdots, X_{m}\right)$ with respect to the last column and divide it by the Vandermonde determinant $D_{0}\left(X_{1}, \cdots, X_{m-1}\right)=D^{0}\left(X_{1}, \cdots, X_{m-1}\right)$. Then

$$
\begin{aligned}
& D_{0}\left(X_{1}, \cdots, X_{m}\right) / D_{0}\left(X_{1}, \cdots, X_{m-1}\right) \\
& \quad=\sum_{j=0}^{m-1}(-1)^{m+(m-1-j+1)} \cdot D^{j} \cdot X_{m}{ }^{m-1-j} / D^{0} \\
& \quad=\sum_{j=0}^{m-1}(-1)^{i} \cdot\left(D^{j} / D^{0}\right) \cdot X_{m}{ }^{m-1-j}
\end{aligned}
$$

Since the Vandermonde determinants are represented as the difference products, we have 


$$
\begin{aligned}
& D_{0}\left(X_{1}, \cdots, X_{m}\right) / D_{0}\left(X_{1}, \cdots, X_{m-1}\right)=\prod_{j=1}^{m-1}\left(X_{m}-X_{j}\right) \\
& \quad=\sum_{j=0}^{m-1}(-1)^{j} \cdot S_{j}\left(X_{1}, \cdots, X_{m-1}\right) \cdot X_{m}^{m-1-j} .
\end{aligned}
$$

Comparing the coefficients of (2.3) and those of (2.4), we have (2.1).

Expand $D_{m}\left(X_{1}, \cdots, X_{m} ; \phi_{1}, \cdots, \phi_{m}\right)$ with respect to the last column and divide it by $D_{0}\left(X_{1}, \cdots, X_{m-1}\right)$. Then

$$
\begin{gathered}
D_{m}\left(X_{1}, \cdots, X_{m} ; \phi_{1}, \cdots, \phi_{m}\right) / D_{0}\left(X_{1}, \cdots, X_{m-1}\right) \\
=\sum_{j=0}^{m-1}(-1)^{j} \cdot S_{j}\left(X_{1}, \cdots, X_{m-1}\right) \cdot \phi_{m-j} .
\end{gathered}
$$

Thus (2.4) and (2.5) imply (2.2) when $k=m$. Since

$$
\begin{aligned}
& D_{k}\left(X_{1}, \cdots, X_{m} ; \phi_{1}, \cdots, \phi_{m}\right) / D_{0}\left(X_{1}, \cdots, X_{m}\right) \\
& \quad=\frac{D_{m}\left(X_{1}, \cdots, X_{k-1}, X_{k+1}, \cdots, X_{m}, X_{k} ; \phi_{1}, \cdots, \phi_{m}\right)}{D_{0}\left(X_{1}, \cdots, X_{k-1}, X_{k+1}, \cdots, X_{m}, X_{k}\right)}
\end{aligned}
$$

we have (2.2) when $k=1, \cdots, m-1$.

Q.E.D.

Let $m=m^{\prime}+m^{\prime \prime}$, where $1 \leqq m^{\prime} \leqq m-1$. When $m^{\prime \prime} \geqq 2$, we put $Y_{j}=X_{m^{\prime}+j}$. $j=1, \cdots, m^{\prime \prime}-1$. When $m^{\prime \prime}=1$, we put

$$
\begin{gathered}
S_{0}\left(Y_{1}, \cdots, Y_{m^{\prime \prime}-1}\right)=1 ; \\
S_{k}\left(Y_{1}, \cdots, Y_{m^{\prime \prime}-1}\right)=0, \text { when } k \neq 0 .
\end{gathered}
$$

Then we have the following lemma.

Lemma 2.3. For $k=1, \cdots, m-1$,

$$
\begin{aligned}
& S_{k}\left(X_{1}, \cdots, X_{m-1}\right)=S_{k}\left(X_{1}, \cdots, X_{m^{\prime}}, Y_{1}, \cdots, Y_{m^{\prime \prime}-1}\right) \\
& \quad=\sum_{j=0}^{m^{\prime \prime}-1} S_{k-j}\left(X_{1}, \cdots, X_{m^{\prime}}\right) \cdot S_{j}\left(Y_{1}, \cdots, Y_{m^{\prime \prime}-1}\right) .
\end{aligned}
$$

Proof. When $m^{\prime \prime}=1,(2.7)$ is trivial. We may assume that $m^{\prime \prime} \geqq 2$. We can write

$$
\begin{aligned}
& S_{k}\left(X_{1}, \cdots, X_{m-1}\right)=\sum_{i_{1}<i_{2}<\cdots<i_{k}} X_{i_{1}} X_{i_{2}} \cdots X_{i_{k}} \\
& \quad=\sum_{j=0}^{m^{\prime \prime}-1} \sum_{i_{1}<i_{2}<\cdots<i_{k-j} \leqq m^{\prime}<i_{k-j+1}<i_{k-j+2}<\cdots<i_{k}} X_{i_{1}} X_{i_{2}} \cdots X_{i_{k}} .
\end{aligned}
$$

Here if $k-j \leqq-1$ or $k-j \geqq m^{\prime}+1$ in the suffixes, then we ignore all such terms. Put $i_{k-j+l}=i_{l}^{\prime}, l=1, \cdots, j$. Then

$$
\begin{aligned}
& S_{k}\left(X_{1}, \cdots, X_{m^{\prime}}, Y_{1}, \cdots, Y_{m^{\prime \prime}-1}\right) \\
& \quad=\sum_{j=0}^{m^{\prime \prime}-1} \sum_{i_{3}<\cdots<i_{k-j} \leqq m^{\prime}, i_{1}^{\prime}<\cdots<i_{j}^{\prime} \leqq m^{\prime \prime}-1} X_{i_{1}} \cdots X_{i_{k-j}} Y_{i_{1}^{\prime}} \cdots Y_{i_{j}^{\prime}} .
\end{aligned}
$$

Since 


$$
\begin{aligned}
& \sum_{i_{1}<\cdots<i_{k-j} \leqq m^{\prime}, i_{1}^{\prime}<\cdots<i_{j}^{\prime} \leqq m^{\prime \prime}-1} X_{i_{1}} \cdots X_{i_{k-j}} Y_{i_{1}^{\prime}} \cdots Y_{i_{j}^{\prime}} \\
& \quad=\left(\sum_{i_{1}<\cdots<i_{k-j} \leqq m^{\prime}} X_{i_{1}} \cdots X_{i_{k-j}}\right)\left(\sum_{i_{1}^{\prime}<\cdots<i_{j}^{\prime} \leqq m^{\prime \prime}-1} Y_{i_{1}^{\prime}} \cdots Y_{i_{j}^{\prime}}\right) \\
& \quad=S_{k-j}\left(X_{1}, \cdots, X_{m^{\prime}}\right) \cdot S_{j}\left(Y_{1}, \cdots, Y_{m^{\prime \prime}-1}\right),
\end{aligned}
$$

it implies that (2.7).

Lemma 2.4. For $k=0, \cdots, m-m^{\prime}-1$ and $l=m^{\prime}+1, \cdots, m$,

$$
\begin{aligned}
& D^{\left(m-m^{\prime}-1\right)-k}\left(\tau_{m^{\prime}+1}^{\prime}, \cdots, \tau_{m-1}^{\prime}\right) \\
& \quad=\prod_{0 \leqq i<j \leqq m-m^{\prime}-1, i \neq k, j \neq k}\left(\zeta^{j}-\zeta^{i}\right)
\end{aligned}
$$

and

$$
\begin{aligned}
& D^{\left(m-m^{\prime}-1\right)-k}\left(\tau_{m^{\prime}+1}^{\prime}, \cdots, \tau_{l-1}^{\prime}, \tau_{l+1}^{\prime}, \cdots, \tau_{m}^{\prime}\right) \\
& \quad=(-1)^{(m-l)^{2}} \cdot \zeta^{k(m-l)} \cdot D^{\left(m-m^{\prime}-1\right)-k}\left(\tau_{m^{\prime}+1}^{\prime}, \cdots, \tau_{m-1}^{\prime}\right) .
\end{aligned}
$$

In particular, $(2.8) \neq 0$ and $(2.9) \neq 0$.

Proof. Put

$$
a_{j}=\left(\tau_{m^{\prime}+1}^{\prime}, \cdots, \tau_{m-1}^{\prime}\right), 0 \leqq j \leqq m-m^{\prime}-1 \text { and } j \neq k
$$

Since

$$
a_{j}=\left(\left(\zeta^{0}\right)^{j}, \cdots,\left(\zeta^{m-m^{\prime}-2}\right)^{j}\right)=\left(\left(\zeta^{j}\right)^{0}, \cdots,\left(\zeta^{j}\right)^{m-m^{\prime}-2}\right),
$$

we have

$$
D^{\left(m-m^{\prime}-1\right)-k}\left(\tau_{m^{\prime}+1}^{\prime}, \cdots, \tau_{m-1}^{\prime}\right)=D_{0}\left(\zeta^{0}, \cdots, \zeta^{k-1}, \zeta^{k+1}, \cdots, \zeta^{m-m^{\prime}-1}\right),
$$

which equals the right-hand side of (2.8).

Multiply the rows $\left(\tau_{m^{\prime}+1}^{\prime}, \cdots, \tau_{l-1}^{\prime}, \tau_{l+1}^{\prime}, \cdots, \tau_{m}^{\prime}\right)$ of the left-hand side of (2.9) by $\zeta^{(m-l) j}, j=1, \cdots, m-m^{\prime}-1$ and $j \neq k$. Then

$$
\begin{aligned}
& \zeta^{(m-l)\left\{\left(m-m^{\prime}-1\right)\left(m-m^{\prime}\right) / 2-k\right\}} \cdot D^{\left(m-m^{\prime}-1\right)-k}\left(\tau_{m^{\prime}+1}^{\prime}, \cdots, \tau_{l-1}^{\prime}, \tau_{l+1}^{\prime}, \cdots, \tau_{m}^{\prime}\right) \\
& \quad=D^{\left(m-m^{\prime}-1\right)-k}\left(\tau_{m+m^{\prime}-l+1}^{\prime}, \cdots, \tau_{m-1}^{\prime}, \tau_{m^{\prime}+1}^{\prime}, \cdots, \tau_{m+m^{\prime}-l}^{\prime}\right) \\
& =(-1)^{\left(l-m^{\prime}-1\right)(m-l)} \cdot D^{\left(m-m^{\prime}-1\right)-k}\left(\tau_{m^{\prime}+1}^{\prime}, \cdots, \tau_{m-1}^{\prime}\right) .
\end{aligned}
$$

Since

$$
\zeta^{(m-l)\left(m-m^{\prime}-1\right)\left(m-m^{\prime}\right) / 2}=(-1)^{(m-l)\left(m-m^{\prime}-1\right)}
$$

and

$$
\left(l-m^{\prime}-1\right)(m-l)-(m-l)\left(m-m^{\prime}-1\right)=-(m-l)^{2} \equiv(m-l)^{2}(\bmod 2),
$$
it implies that (2.9). 
Under Assumption 1.1, we have studied the following asymptotic properties of the characteristic roots in [2]. Denote the characteristic roots of $\varepsilon^{m-m^{\prime}}$. $P_{1}(\xi)+P_{2}(\xi)=0$ with respect to $\xi_{1}$ by $\tau_{j}\left(\varepsilon, \xi^{\prime}\right), j=1, \cdots, m$ and those of $P_{2}(\xi)=0$ by $\sigma_{j}\left(\xi^{\prime}\right), j=1, \cdots, m^{\prime}$.

Lemma 2.5. Let Assumption 1.1 be satisfied and $B_{0}$ be the open ball in Remark to Assumption 1.1. If the suffixes $\{j\}$ of the characteristic roots $\tau_{j}\left(\varepsilon, \xi^{\prime}\right)$, $j=1, \cdots, m$ are properly chosen, then there exists a positive number $\varepsilon_{0}$ such that $\varepsilon \cdot \tau_{j}\left(\varepsilon, \xi^{\prime}\right), j=1, \cdots, m$ are analytic functions of $\left(\varepsilon, \xi^{\prime}\right)$ in $\left\{|\varepsilon|<\varepsilon_{0}\right\} \times B_{0}$ and $\tau_{j}\left(\varepsilon, \xi^{\prime}\right), j=1, \cdots, m$ have the following representations:

For $j=1, \cdots, m^{\prime}$

$$
\tau_{j}\left(\varepsilon, \xi^{\prime}\right)=\sigma_{j}\left(\xi^{\prime}\right)+\sum_{k=2}^{\infty} S_{j, k}\left(\xi^{\prime}\right) \varepsilon^{(k-1)\left(m-m^{\prime}\right)},
$$

where $\partial_{1}=\frac{\partial}{\partial \xi_{1}}$ and

$$
s_{j, 2}=-P_{1}\left(\sigma_{j}, \xi^{\prime}\right) \cdot \partial_{1} P_{2}\left(\sigma_{j}, \xi^{\prime}\right)^{-1}
$$

For $j=m^{\prime}+1, \cdots, m$

$$
\tau_{j}\left(\varepsilon, \xi^{\prime}\right)=\Theta \tau_{j}^{\prime} \cdot \frac{1}{\varepsilon}+\sum_{k=2}^{\infty} t_{j, k}\left(\xi^{\prime}\right) \cdot \varepsilon^{k-2} .
$$

Remark. If $s_{j, 2}\left(\xi^{\prime}\right) \equiv 0$ in $B_{0}$, then $P_{1}\left(\sigma_{j}\left(\xi^{\prime}\right), \xi^{\prime}\right) \equiv 0$ in $B_{0}$. This implies that $\tau_{j}\left(\varepsilon, \xi^{\prime}\right) \equiv \sigma_{j}\left(\xi^{\prime}\right)$ in $B_{0}$.

\section{The Condition for the Convergence of the Solutions}

In this section, we shall study a necessary and sufficient condition for the convergence when Condition 1.2 is not satisfied. The main result in this section is the following:

Proposition. Assume that Condition 1.2 is not satisfied. Let Assumption 1.1 be satisfied and $B_{0}$ be the open ball in Remark to Assumption 1.1. Let the Cauchy data $\Phi=\left(\phi_{1}, \cdots, \phi_{m}\right)$ of $(1.1)$ belong to $\mathrm{F}^{-1}\left(C_{0}^{\infty}\left(B_{0}\right)\right)^{m}$. The solutions $u_{\mathrm{g}}(x ; \Phi)$ of $(1.1)$ converge in $C\left(\mathbb{R}^{n}\right)$ if and only if the Cauchy data $\Phi$ satisfy

$$
\sum_{k=0}^{m^{\prime}}(-1)^{k} \cdot S_{k}\left(\tau_{1}, \cdots, \tau_{m^{\prime}}\right) \cdot \hat{\phi}_{m^{\prime}+j-k} \equiv 0, j=1, \cdots, m-m^{\prime} .
$$

Here $S_{k}\left(\tau_{1}, \cdots, \tau_{m^{\prime}}\right), k=0, \cdots, m^{\prime}$ are the elementary symmetric polynomials of the characteristic roots $\tau_{j}=\tau_{j}\left(\varepsilon, \xi^{\prime}\right), j=1, \cdots, m^{\prime}$ mentioned in Notation 2.1. For the Cauchy data satisfying (3.1), the partial Fourier transforms of solutions $u_{\mathrm{z}}(x ; \Phi)$ of $(1.1)$ are 


$$
\hat{u}_{\varepsilon}\left(x_{1}, \xi^{\prime}\right)=\sum_{j=1}^{m^{\prime}} \frac{D_{j}\left(\tau_{1}, \cdots, \tau_{m} ; \hat{\phi}_{1}, \cdots, \hat{\phi}_{m}\right)}{D_{0}\left(\tau_{1}, \cdots, \tau_{m}\right)} \cdot \exp i \tau_{j}\left(\varepsilon, \xi^{\prime}\right) x_{1} \text {. }
$$

Remark. Denote $\hat{\Phi}=\left(\hat{\phi}_{1}, \cdots, \hat{\phi}_{m}\right)$. Let $\beta$ belong to $C^{\infty}\left(B_{0}\right)$. If $\hat{\Phi}$ satisfies (3.1), then $\beta \cdot \hat{\Phi}$ also satisfies (3.1). Hence if there exists a Cauchy datum $\Phi_{0}$ such that the solutions $u_{\varepsilon}\left(x ; \Phi_{0}\right)$ converge, then for all the Cauchy data $\Phi$ satisfying $\hat{\Phi}=\beta \cdot \hat{\Phi}_{0}$, where $\beta$ belongs to $C^{\infty}\left(B_{0}\right)$, the solutions $u_{\varepsilon}(x ; \Phi)$ converge.

In order to prove Proposition, we need the following lemma. Denote $L=$ $\left\{l ; \operatorname{Im} \Theta \tau_{l}^{\prime} \neq 0, l=m^{\prime}+1, \cdots, m\right\}$, where $\Theta$ and $\tau_{l}^{\prime}, l=m^{\prime}+1, \cdots, m$ are mentioned in Notation 2.1.

Lemma 3.1. Let the same assumptions as in Proposition be satisfied. The solutions $u_{\varepsilon}(x ; \Phi)$ of $(1.1)$ converge in $C\left(\boldsymbol{R}^{n}\right)$ if and only if the Cauchy data $\Phi$ satisfy

$$
\begin{aligned}
& \varepsilon^{m-1} \cdot \sum_{j=0}^{m-1}(-1)^{j} \cdot S_{j}\left(\tau_{1}, \cdots, \tau_{l-1}, \tau_{l+1}, \cdots, \tau_{m}\right) \cdot \hat{\phi}_{m-j} \\
& \quad=\sum_{j=0}^{m-1}(-1)^{j} \cdot \varepsilon^{m-1-j} \cdot S_{j}\left(\varepsilon \tau_{1}, \cdots, \varepsilon \tau_{l-1}, \varepsilon \tau_{l+1}, \cdots, \varepsilon \tau_{m}\right) \cdot \hat{\phi}_{m-j} \equiv 0
\end{aligned}
$$

as analytic functions of $\varepsilon$ for all $l$ in $L$.

Proof. The partial Fourier transform with respect to $x^{\prime}$ of (1.1) is

$$
\left\{\begin{array}{l}
\left(\varepsilon^{m-m^{\prime}} \cdot P_{1}\left(D_{1}, \xi^{\prime}\right)+P_{2}\left(D_{1}, \xi^{\prime}\right)\right) \hat{u}\left(x_{1}, \xi^{\prime}\right)=0 ; \\
D_{1}^{j-1} \hat{u}\left(0, \xi^{\prime}\right)=\hat{\phi}_{j}\left(\xi^{\prime}\right), j=1, \cdots, m .
\end{array}\right.
$$

For fixed $\xi^{\prime},(3.4)$ is a one-parameter family of Cauchy problems of ordinary differential equations. For $\tau_{j}\left(\varepsilon, \xi^{\prime}\right), j=1, \cdots, m$ satifying Lemma 2.5 and for $\Phi$ in $\mathrm{F}^{-1}\left(C_{0}^{\infty}\left(B_{0}\right)\right)^{m}$, put

$$
C_{k}\left(\varepsilon, \xi^{\prime} ; \Phi\right)=D_{k}\left(\tau_{1}, \cdots, \tau_{m} ; \hat{\phi}_{1}, \cdots, \hat{\phi}_{m}\right) / D_{0}\left(\tau_{1}, \cdots, \tau_{m}\right),
$$

$k=1, \cdots, m$. Since (3.5) are analytic in $\varepsilon$ for sufficiently small $\varepsilon$, (3.5) have power series representations as

$$
C_{k}\left(\varepsilon, \xi^{\prime} ; \Phi\right)=\sum_{j=0}^{\infty} c_{k, j}\left(\xi^{\prime} ; \Phi\right) \cdot \varepsilon^{j}, k=1, \cdots, m .
$$

The solution $v_{\varepsilon}\left(x_{1}, \xi^{\prime}\right)$ of (3.4) is represented as

$$
v_{\varepsilon}\left(x_{1}, \xi^{\prime}\right)=\sum_{k=1}^{m} C_{k}\left(\varepsilon, \xi^{\prime} ; \Phi\right) \cdot \exp i \tau_{k}\left(\varepsilon, \xi^{\prime}\right) x_{1} .
$$

Denote $u_{\varepsilon}(x)=\mathrm{F}^{-1}\left(v_{\varepsilon}\left(x_{1}, \xi^{\prime}\right)\right)$. By the same argument as in [3], we can prove that if there exist integers $l$ in $L$ and $j$ with $j \geqq 0$ such that $c_{l, j}\left(\xi^{\prime} ; \Phi\right) \neq 0$, then $u_{\varepsilon}(x)$ can not converge in $C\left(\boldsymbol{R}^{n}\right)$. When Condition 1.2 is not satisfied, $L$ is not void. Therefore it is necessary for the convergence of $u_{\mathrm{e}}(x)$ in $C\left(\boldsymbol{R}^{n}\right)$ that 
$c_{l, j}\left(\xi^{\prime} ; \Phi\right) \equiv 0$ for all $l$ in $L$ and all $j \geqq 0$, that is, $C_{l}\left(\varepsilon, \xi^{\prime} ; \Phi\right) \equiv 0$ for all $l$ in $L$ and sufficiently small $\varepsilon$, which implies the convergence of solutions. Since

$$
\tau_{j}\left(\varepsilon, \xi^{\prime}\right) \neq \tau_{k}\left(\varepsilon, \xi^{\prime}\right), \quad 1 \leqq j<k \leqq m
$$

in $B_{0}$ for sufficiently small $\varepsilon$, we can apply Lemma 2.2 to $C_{l}\left(\varepsilon, \xi^{\prime} ; \Phi\right)$ for $X_{j}=$ $\tau_{j}\left(\varepsilon, \xi^{\prime}\right), j=1, \cdots, m$. Then

$$
C_{l}=\frac{\varepsilon^{m-1} \cdot \sum_{j=0}^{m-1}(-1)^{j} \cdot S_{j}\left(\tau_{1}, \cdots, \tau_{l-1}, \tau_{l+1}, \cdots, \tau_{m}\right) \cdot \hat{\phi}_{m-j}}{\varepsilon^{m-1} \cdot \sum_{j=0}^{m-1}(-1)^{j} \cdot S_{j}\left(\tau_{1}, \cdots, \tau_{l-1}, \tau_{l+1}, \cdots, \tau_{m}\right) \cdot \tau_{l}^{m-1-j}} .
$$

The denominators are equal to

$$
\begin{aligned}
& \sum_{j=0}^{m-1}(-1)^{j} \cdot S_{j}\left(\varepsilon \tau_{1}, \cdots, \varepsilon \tau_{l+1}, \varepsilon \tau_{l+1}, \cdots, \varepsilon \tau_{m}\right) \cdot\left(\varepsilon \tau_{l}\right)^{m-1-j} \\
& \quad=\prod_{1 \leqq j \leqq m, j \neq l}\left(\varepsilon \tau_{l}-\varepsilon \tau_{j}\right)
\end{aligned}
$$

and analytic in $\varepsilon$ and not zero in $B_{0}$ for sufficiently small $\varepsilon$. This implies that $C_{l}\left(\varepsilon, \xi^{\prime} ; \Phi\right) \equiv 0$ if and only if the numerators

$$
\begin{aligned}
& \varepsilon^{m-1} \cdot \sum_{j=0}^{m-1}(-1)^{j} \cdot S_{j}\left(\tau_{1}, \cdots, \tau_{l-1}, \tau_{l+1}, \cdots, \tau_{m}\right) \cdot \hat{\phi}_{m-j} \\
& \quad=\sum_{j=0}^{m-1}(-1)^{j} \cdot \varepsilon^{m-1-j} \cdot S_{j}\left(\varepsilon \tau_{1}, \cdots, \varepsilon \tau_{l-1}, \varepsilon \tau_{l+1}, \cdots, \varepsilon \tau_{m}\right) \cdot \hat{\phi}_{m-j}
\end{aligned}
$$

are zero as analytic functions of $\varepsilon$. Thus we come to the conclusion. Q.E.D.

Proof of Proposition. Fix $l$ in $L$ and apply Lemma 2.3 to (3.3) for

$$
\begin{array}{cl}
X_{j}=\tau_{j}\left(\varepsilon, \xi^{\prime}\right), & j=1, \cdots, m^{\prime} . \\
Y_{j-m^{\prime}}=\tau_{j}\left(\varepsilon, \xi^{\prime}\right), & j=m^{\prime}+1, \cdots, l-1, \\
Y_{j-m^{\prime}-1}=\tau_{j}\left(\varepsilon, \xi^{\prime}\right), & j=l+1, \cdots, m .
\end{array}
$$

Then the convergence of solutions is equivalent to

$$
\begin{gathered}
\varepsilon^{m-1} \cdot \sum_{j=0}^{m-1}(-1)^{j} \cdot S_{j}\left(\tau_{1}, \cdots, \tau_{l-1}, \tau_{l+1}, \cdots, \tau_{m}\right) \cdot \hat{\phi}_{m-j} \\
=\varepsilon^{m-1} \cdot \sum_{j=0}^{m-1}(-1)^{j} \cdot\left(\sum_{k=0}^{m-m-1} S_{j-k}\left(\tau_{1}, \cdots, \tau_{m^{\prime}}\right)\right. \\
\left.\cdot S_{k}\left(\tau_{m^{\prime}+1}, \cdots, \tau_{l-1}, \tau_{l+1}, \cdots, \tau_{m}\right) \cdot \hat{\phi}_{m-j}\right) \equiv 0 .
\end{gathered}
$$

Substitute (2.10) and (2.13) for $\tau_{j}\left(\varepsilon, \xi^{\prime}\right), j=1, \cdots, m$. Then we can write for $j=1, \cdots, m-1$ and $k=0, \cdots, m-m^{\prime}-1$

$$
\begin{gathered}
S_{j-k}\left(\tau_{1}, \cdots, \tau_{m^{\prime}}\right)=\sum_{i=0}^{\infty} S_{j-k, i}^{1}\left(\xi^{\prime}\right) \cdot \varepsilon^{i\left(m-m^{\prime}\right)} \\
\varepsilon^{k} \cdot S_{k}\left(\tau_{m^{\prime}+1}, \cdots, \tau_{l-1}, \tau_{l+1}, \cdots, \tau_{m}\right) \\
=S_{k}\left(\varepsilon \tau_{m^{\prime}+1}, \cdots, \varepsilon \tau_{l-1}, \varepsilon \tau_{l+1}, \cdots, \varepsilon \tau_{m}\right)=\sum_{i=0}^{\infty} S_{k, i}^{2}\left(\xi^{\prime}\right) \cdot \varepsilon^{i} .
\end{gathered}
$$

Here (3.8) and (3.9) are analytic and converge absolutely for sufficiently small 
$\varepsilon$ in $B_{0}$ and

$$
\begin{gathered}
S_{j-k, 0}^{1}\left(\xi^{\prime}\right)=S_{j-k}\left(\sigma_{1}, \cdots, \sigma_{m^{\prime}}\right), \\
S_{k, 0}^{2}\left(\xi^{\prime}\right)=\Theta^{k} \cdot S_{k}\left(\tau_{m^{\prime}+1}^{\prime}, \cdots, \tau_{l-1}^{\prime}, \tau_{l+1}^{\prime}, \cdots, \sigma_{m}^{\prime}\right) .
\end{gathered}
$$

Put for $\kappa=0,1, \cdots$

$$
\begin{aligned}
& T_{1, \kappa}=\sum_{j=0}^{m-1}(-1)^{j} \cdot \sum_{k=0}^{m-m^{\prime}-1} S_{j-k, \kappa}^{1} \cdot \varepsilon^{k\left(m-m^{\prime}\right)} \cdot S_{k, 0}^{2} \cdot \varepsilon^{m-1-k} \cdot \hat{\phi}_{m-j}, \\
& T_{2, \kappa}=\sum_{j=0}^{m-1}(-1)^{j} \cdot \sum_{k=0}^{m-m^{\prime}-1} S_{j-k, \kappa}^{1} \cdot \varepsilon^{\kappa\left(m-m^{\prime}\right)} \cdot\left(\sum_{i=1}^{\infty} S_{k, i}^{2} \cdot \varepsilon^{m-1-k+i}\right) \cdot \hat{\phi}_{m-j},
\end{aligned}
$$

and

$$
T_{3, \mathrm{\kappa}}=\sum_{j=0}^{m-1}(-1)^{j} \cdot \sum_{k=0}^{m-m^{\prime}-1}\left(\sum_{i=\kappa+1}^{\infty} S_{j-k, i}^{1} \cdot \varepsilon^{i\left(m-m^{\prime}\right)}\right) \cdot\left(\sum_{i=0}^{\infty} S_{k, i}^{2} \cdot \varepsilon^{m-1-k+i}\right) \cdot \hat{\phi}_{m-j} .
$$

Since $T_{2, \kappa}$ and $T_{3, \kappa}$ converge absolutely for sufficiently small $\varepsilon$, we can change the order of summations. Then (3.7) is rewritten as $T_{1,0}+T_{2,0}+T_{3,0} \equiv 0$. The least order with respect to $\varepsilon$ of $T_{1,0}$ is $m^{\prime}$, that of $T_{2,0}$ is $m^{\prime}+1$, and that of $T_{3,0}$ is $m$. The coefficient of $\varepsilon^{m^{\prime}}$ in $T_{1,0}$ is

$$
\begin{aligned}
& \sum_{j=0}^{m-1}(-1)^{j} \cdot S_{j-\left(m-m^{\prime}-1\right), 0}^{1} \cdot S_{m-m^{\prime}-1,0}^{2} \cdot \hat{\phi}_{m-j} \\
& \quad=S_{m-m^{\prime}-1,0}^{2} \cdot \sum_{j=m-m^{\prime}-1}^{m-1}(-1)^{j} \cdot S_{j-\left(m-m^{\prime}-1\right), 0}^{1} \cdot \hat{\phi}_{m-j}
\end{aligned}
$$

and this must be zero. Lemma 2.2 implies that

$$
\begin{gathered}
S_{m-m^{\prime}-1,0}^{2}=\Theta^{m-m^{\prime}-1} \cdot S_{m-m^{\prime}-1}\left(\tau_{m^{\prime}+1}^{\prime}, \cdots, \tau_{l-1}^{\prime}, \tau_{l+1}^{\prime}, \cdots, \tau_{m}^{\prime}\right) \\
=\Theta^{m-m^{\prime}-1} \times \frac{D^{\left(m-m^{\prime}-1\right)-0}\left(\tau_{m^{\prime}+1}^{\prime}, \cdots, \tau_{l-1}^{\prime}, \tau_{l+1}^{\prime}, \cdots, \tau_{m}^{\prime}\right)}{D^{0}\left(\tau_{m^{\prime}+1}^{\prime}, \cdots, \tau_{l-1}^{\prime}, \tau_{l+1}^{\prime}, \cdots, \tau_{m}^{\prime}\right)} .
\end{gathered}
$$

Lemma 2.4 implies that

$$
\frac{D^{\left(m-m^{\prime}-1\right)-0}\left(\tau_{m^{\prime}+1}^{\prime}, \cdots, \tau_{l-1}^{\prime}, \tau_{l+1}^{\prime}, \cdots, \tau_{m}^{\prime}\right)}{D^{0}\left(\tau_{m^{\prime}+1}^{\prime}, \cdots, \tau_{l-1}^{\prime}, \tau_{l+1}^{\prime}, \cdots, \tau_{m}^{\prime}\right)} \neq 0
$$

Hence $S_{m-m^{\prime}-1,0}^{2} \neq 0$. Dividing (3.13) by $S_{m-m^{\prime}-1,0}^{2}$, we have

$$
\begin{aligned}
& \sum_{j=m-m^{\prime}-1}^{m-1}(-1)^{j} \cdot S_{j-\left(m-m^{\prime}-1\right), 0}^{1} \cdot \hat{\phi}_{m-j} \\
& \quad=(-1)^{m-m^{\prime}-1} \cdot \sum_{j=0}^{m^{\prime}}(-1)^{j} \cdot S_{j, 0}^{1} \cdot \hat{\phi}_{m^{\prime}+1-j}=0 .
\end{aligned}
$$

When (3.14) is satisfied, we have

$$
T_{1,0}=\sum_{j=0}^{m-1}(-1)^{j} \cdot \sum_{k=0}^{m-m^{\prime}-2}\left(S_{j-k, 0}^{1} \cdot S_{k, 0}^{2} \cdot \varepsilon^{m-1-k} \cdot \hat{\phi}_{m-j}\right)
$$

and

$$
\begin{aligned}
& T_{2,0}=\sum_{j=0}^{m-1}(-1)^{j} \cdot S_{j-\left(m-m^{\prime}-1\right), 0}^{1} \cdot\left(\sum_{i=1}^{\infty} S_{m-m^{\prime}-1, i}^{2} \cdot \varepsilon^{m^{\prime}+i}\right) \cdot \hat{\phi}_{m-j} \\
& \quad+\sum_{j=0}^{m-1}(-1)^{j} \cdot \sum_{k=0}^{m-m^{\prime}-2} S_{j-k, 0}^{1} \cdot\left(\sum_{i=1}^{\infty} S_{k, i}^{2} \cdot \varepsilon^{m-1-k+i}\right) \cdot \hat{\phi}_{m-j}
\end{aligned}
$$




$$
\begin{aligned}
& =\sum_{i=1}^{\infty} S_{m-m^{\prime}-1, i}^{2} \cdot \varepsilon^{m^{\prime}+i} \cdot\left(\sum_{j=0}^{n-1}(-1)^{j} \cdot S_{j-\left(m-m^{\prime}-1\right), 0}^{1} \hat{\phi}_{m-j}\right) \\
& +\sum_{j=0}^{m-1}(-1)^{j} \cdot \sum_{k=0}^{m-m^{\prime}-2} S_{j-k, 0}^{1} \cdot\left(\sum_{i=1}^{\infty} S_{k, i}^{2} \cdot \varepsilon^{m-1-k+i}\right) \hat{\phi} \cdot{ }_{m-j} \\
& =\sum_{j=0}^{n-1}(-1)^{j} \cdot \sum_{k=0}^{m-m^{\prime}-2} S_{j-k, 0}^{1} \cdot\left(\sum_{i=1}^{\infty} S_{k, 2}^{2} \cdot \varepsilon^{m-1-k+i}\right) \cdot \hat{\phi}_{m-j} .
\end{aligned}
$$

Thus we can diminish by one the ranges of summations with respect to $k$ in $T_{1,0}$ and in $T_{2,0}$. Repeat this process $m-m^{\prime}-1$ times until the ranges of summations with respect to $k$ are diminished to $\{0\}$. Then we have for $j=1, \cdots$, $m-m^{\prime}$

$$
\sum_{k=0}^{m^{\prime}}(-1)^{k} \cdot S_{k, 0}^{1} \cdot \hat{\phi}_{m^{\prime}+j-k}=0
$$

and $T_{1,0} \equiv T_{2,0} \equiv 0$. Hence

$$
T_{1,0}+T_{2,0}+T_{3,0}=T_{3,0}=T_{1,1}+T_{2,1}+T_{3,1} \equiv 0 .
$$

Repeating the above argument for $\kappa=1,2, \cdots$, we have for $k=0,1, \cdots$

$$
\sum_{k=0}^{m^{\prime}}(-1)^{k} \cdot S_{k, \kappa}^{1} \cdot \phi_{m^{\prime}+j-k}^{\wedge}=0, \quad j=1, \cdots, m-m^{\prime},
$$

which are equivalent to (3.3). Multiply (3.16) by $\varepsilon^{k\left(m-m^{\prime}\right)}$ and sum up from $r=0$ to $\kappa=\infty$. Even if we change $l$ in $L$, we have the same conclusion. Hence (3.16) are equivalent to (3.1) as far as (3.8) converge absolutely. Since (3.16) are independent of $l$ in $L$, it implies that (3.3) remain true for all $l=m^{\prime}+$ $1, \cdots, m$ and $C_{l}\left(\varepsilon, \xi^{\prime} ; \Phi\right) \equiv 0$ for all $l=m^{\prime}+1, \cdots, m$. Then (3.6) implies (3.2). Thus we come to the conclusion.

Q.E.D.

Put

$$
\begin{gathered}
\tilde{p}_{2, k}\left(\xi^{\prime}\right)=-p_{2, k}\left(\xi^{\prime}\right) / p_{2,0}, \quad k=0, \cdots, m^{\prime}, \\
\tilde{p}_{2, k}=0, \text { for } k \leqq-1 \text { or } k \geqq m^{\prime}+1 .
\end{gathered}
$$

Here $\tilde{p}_{2, k}\left(\xi^{\prime}\right), k=0, \cdots, m^{\prime}$ are polynomials of $\xi^{\prime}$, because $p_{2,0}$ is a non-zero constant. Denote

$$
\begin{aligned}
& Q\left(\xi^{\prime}\right)=\left(-\tilde{p}_{2, j-k}\left(\xi^{\prime}\right) ; j \downarrow 1, \cdots, m-m^{\prime}, k \rightarrow 1, \cdots, m-m^{\prime}\right), \\
& R\left(\xi^{\prime}\right)=\left(\tilde{p}_{2, m^{\prime}+j-k}\left(\xi^{\prime}\right) ; j \downarrow 1, \cdots, m-m^{\prime}, k \rightarrow 1, \cdots, m^{\prime}\right) .
\end{aligned}
$$

Here $Q\left(\xi^{\prime}\right)$ is a lower triangular matrix all whose diagonal components are 1. Hence $Q^{-1}\left(\xi^{\prime}\right)$ is a matrix with polynomial components. Denote $\hat{\Phi}^{\prime}=$ $\left(\hat{\phi}_{1}, \cdots, \hat{\phi}_{m^{\prime}}\right)$ and $\hat{\Phi}^{\prime \prime}=\left(\hat{\phi}_{m^{\prime}+1}, \cdots, \hat{\phi}_{m}\right)$.

Corollary 3.2. Let the same assumptions as in Proposition be satisfied. Then the convergence of solutions implies that 


$$
\sum_{k=0}^{m^{\prime}} p_{2, k}\left(\xi^{\prime}\right) \cdot \hat{\phi}_{m^{\prime}+j-k}\left(\xi^{\prime}\right)=0, \quad j=1, \cdots, m-m^{\prime} .
$$

Then (3.17) are equivalent to

$$
{ }^{t} \hat{\Phi}^{\prime \prime}=Q^{-1}\left(\xi^{\prime}\right) \cdot R\left(\xi^{\prime}\right) \cdot{ }^{t} \hat{\Phi}^{\prime} .
$$

The inverse Fourier transforms of (3.18) are

$$
{ }^{t} \Phi^{\prime \prime}=Q^{-1}\left(D^{\prime}\right) \cdot R\left(D^{\prime}\right) \cdot{ }^{t} \Phi^{\prime}
$$

Proof. Since

$$
P_{2}(\xi) / p_{2,0}=\xi_{1}^{m^{\prime}}-\sum_{j=1}^{m^{\prime}} \tilde{p}_{2, j}\left(\xi^{\prime}\right) \cdot \xi_{1}^{m^{\prime}-j}=\prod_{j=1}^{m^{\prime}}\left(\xi_{1}-\sigma_{j}\right),
$$

it implies that

$$
-\tilde{p}_{2, j}\left(\xi^{\prime}\right)=(-1)^{j} \cdot S_{j}\left(\sigma_{1}, \cdots, \sigma_{m}\right), \quad j=1, \cdots, m^{\prime} .
$$

Hence (3.10) and (3.15) imply that for $j=1, \cdots, m-m^{\prime}$

$$
\sum_{k=0}^{m^{\prime}} \tilde{p}_{2, k} \cdot \hat{\phi}_{m^{\prime}+j-k}=0,
$$

which is equivalent to (3.17). By (3.20), we have

$$
-\sum_{k=0}^{j-1} \tilde{p}_{2, k} \cdot \phi_{m^{\prime}+j-k}=\sum_{k=j}^{m^{\prime}} \tilde{p}_{2, k} \cdot \hat{\phi}_{m^{\prime}+j-k}, \quad j=1, \cdots, m-m^{\prime},
$$

which are equivalent to (3.18).

Remark. In [3], we used the function $d\left(\xi^{\prime} ; \Phi\right)$ to decide the divergence of solutions. Corollary 3.2 implies that $d\left(\xi^{\prime} ; \Phi\right) \equiv 0$ is equivalent to $\sum_{k=0}^{m \prime^{\prime}} p_{2, k}\left(\xi^{\prime}\right)$ - $\hat{\phi}_{m^{\prime}+1-k}\left(\xi^{\prime}\right) \equiv 0$. We had better use (3.18) instead of $d\left(\xi^{\prime} ; \Phi\right) \equiv 0$ because (3.18) are more accurate and algebraic relations between the coefficients of $P_{2}$ and the Cauchy data.

\section{Denote}

$$
\begin{aligned}
A & =\left(a_{j, k} ; j \downarrow 1, \cdots, m-m^{\prime}, k \rightarrow 1, \cdots, m^{\prime}\right) \\
& =\left(\hat{\phi}_{j+m^{\prime}-k} ; j \downarrow 1, \cdots, m-m^{\prime}, k \rightarrow 1, \cdots, m^{\prime}\right),
\end{aligned}
$$

for $i=1, \cdots, m^{\prime}$

$$
\begin{aligned}
b_{i} & =\left(b_{k, i} ; k \downarrow 1, \cdots, m^{\prime}\right) \\
& =\left((-1)^{k-1} \cdot S_{k-1}\left(\sigma_{1}, \cdots, \sigma_{i-1}, \sigma_{i+1}, \cdots, \sigma_{m^{\prime}}\right) ; k \downarrow 1, \cdots, m^{\prime}\right) .
\end{aligned}
$$

Here we define $S_{0}=1$ even when $m^{\prime}=1$. Denote

$$
\begin{aligned}
& B=\left(b_{i} ; i \rightarrow 1, \cdots, m^{\prime}\right), \\
& s=\left(s_{i, 2} ; i \downarrow 1, \cdots, m^{\prime}\right),
\end{aligned}
$$


where $s_{i, 2}=-P_{1}\left(\sigma_{i}, \xi^{\prime}\right) \cdot \partial_{1} P_{2}\left(\sigma_{i}, \xi^{\prime}\right)^{-1}, i=1, \cdots, m^{\prime}$. Then we have the following corollary.

Corollary 3.3. Let the same assumptions as in Proposition be satisfied. Then det $B=\mathbb{I}_{1 \leqq i<j \leqq m^{\prime}}\left(\sigma_{j}-\sigma_{\dot{i}}\right) \neq 0$ in $B_{0}$. The convergence of the solutions implies that $A B s=0$, which are equivalent to

$$
\begin{gathered}
\sum_{j=1}^{m^{\prime}} S_{i, 2} \sum_{k=0}^{m^{\prime}-1}(-1)^{k} \cdot S_{k}\left(\sigma_{1}, \cdots, \sigma_{i-1}, \sigma_{i+1}, \cdots, \sigma_{m^{\prime}}\right) \\
\cdot \hat{\phi}_{m^{\prime}-1+j-k}=0, \quad j=1, \cdots, m-m^{\prime} .
\end{gathered}
$$

Proof. Since for $k=1, \cdots, m^{\prime}-1$ and $i=1, \cdots, m^{\prime}-1$,

$$
\begin{aligned}
& S_{k}\left(\sigma_{1}, \cdots, \sigma_{i-1}, \sigma_{i+1}, \cdots, \sigma_{m^{\prime}}\right)=\sigma_{m^{\prime}} \cdot S_{k-1}\left(\sigma_{1}, \cdots \sigma_{i-1}, \sigma_{i+1}, \cdots \sigma_{m^{\prime}-1}\right) \\
& \quad+S_{k}\left(\sigma_{1}, \cdots, \sigma_{i-1}, \sigma_{i+1}, \cdots \sigma_{m^{\prime}-1}\right)
\end{aligned}
$$

and

$$
\begin{aligned}
& S_{k}\left(\sigma_{1}, \cdots, \sigma_{m^{\prime}-1}\right)=\sigma_{i} \cdot S_{k-1}\left(\sigma_{1}, \cdots, \sigma_{i-1}, \sigma_{i+1}, \cdots \sigma_{m^{\prime}-1}\right) \\
& \quad+S_{k}\left(\sigma_{1}, \cdots, \sigma_{i-1}, \sigma_{i+1}, \cdots \sigma_{m^{\prime}-1}\right),
\end{aligned}
$$

it implies that for $i=1, \cdots, m^{\prime}-1$

$$
\begin{aligned}
& b_{i}-b_{m^{\prime}}=\left(\sigma_{i}-\sigma_{m^{\prime}}\right) \\
& \quad \times\left((-1)^{k-2} \cdot S_{k-2}\left(\sigma_{1}, \cdots, \sigma_{i-1}, \sigma_{i+1}, \cdots \sigma_{m^{\prime}-1}\right) ; k \downarrow 1, \cdots, m-m^{\prime}\right),
\end{aligned}
$$

where $S_{-1}=0$. Then

$$
\begin{aligned}
\operatorname{det} & \left(b_{1}, \cdots, b_{m^{\prime}}\right)=\operatorname{det}\left(b_{1}-b_{m^{\prime}}, \cdots, b_{m^{\prime}-1}-b_{m^{\prime}}, b_{m^{\prime}}\right) \\
= & (-1)^{m^{\prime}+1} \cdot \prod_{i=1}^{m^{\prime}-1}\left(\sigma_{i}-\sigma_{m^{\prime}}\right) \cdot \operatorname{det}\left(b_{k, i} ; j \downarrow 1, \cdots, m^{\prime}-1, k \rightarrow 1, \cdots, m^{\prime}-1\right) \\
= & \prod_{i=1}^{m^{\prime}-1}\left(\sigma_{m^{\prime}}-\sigma_{i}\right) \cdot \operatorname{det}\left(b_{k, i} ; j \downarrow 1, \cdots, m^{\prime}-1, k \rightarrow 1, \cdots, m^{\prime}-1\right) .
\end{aligned}
$$

Hence det $B$ is equal to the difference product of $\sigma_{j}, j=1, \cdots, m^{\prime}$. Since $\sigma_{j} \neq \sigma_{k}$, $1 \leqq j<k \leqq m^{\prime}$ in $B_{0}$, it implies that det $B \neq 0$ in $B_{0}$. Since $S_{0,1}^{1}=0$ and

$$
S_{k, 1}^{1}=\sum_{i=1}^{m^{\prime}} S_{i, 2} \cdot S_{k-1}\left(\sigma_{1}, \cdots, \sigma_{i-1}, \sigma_{i+1}, \cdots, \sigma_{m}{ }^{\prime}\right), k=1, \cdots, m-m^{\prime},
$$

(3.16) for $\kappa=1$ implies that

$$
\begin{aligned}
& -\sum_{i=1}^{m^{\prime}} S_{i, 2} \sum_{k=1}^{m^{\prime}}(-1)^{k-1} \cdot S_{k-1}\left(\sigma_{1}, \cdots, \sigma_{i-1}, \sigma_{i+1}, \cdots, \sigma_{m^{\prime}}\right) \\
& \quad \times \hat{\phi}_{m^{\prime}+j-k}=0, \quad j=1, \cdots, m-m^{\prime},
\end{aligned}
$$

which are equivalent to $A B s=0$.

Q.E.D.

In the rest of this section, we shall study conditions on the Cauchy data in special forms for the convergence of the solutions. 
Corollary 3.4. Let the same assumptions as in Proposition be satisfied. Let $\Phi^{\prime}=\left(\phi_{1}, \cdots, \phi_{m^{\prime}}\right)=0$. The solutions $u_{\varepsilon}(x ; \Phi)$ of $(1.1)$ converge in $C\left(\boldsymbol{R}^{n}\right)$ for this Cauchy data if and only if $\Phi^{\prime \prime}=\left(\phi_{m^{\prime}+1}, \cdots, \phi_{m}\right)=0$.

Proof. Assume that the solutions converge. Substitute $\Phi^{\prime}=0$ in (3.19). Then $\Phi^{\prime \prime}=0$. The converse is trivial.

Q.E.D.

Corollary 3.5. Let the same assumptions as in Proposition be satisfied. Assume that $m-m^{\prime} \geqq m^{\prime}$ and $p_{2, m^{\prime}} \neq 0$. Let $\Phi^{\prime \prime}=\left(\phi_{m^{\prime}+1}, \cdots, \phi_{m}\right)=0$. The solutions $u_{\varepsilon}(x ; \Phi)$ of $(1.1)$ converge in $C\left(\boldsymbol{R}^{n}\right)$ for this Cauchy data if and only if $\Phi^{\prime}=$ $\left(\phi_{1}, \cdots, \phi_{m^{\prime}}\right)=0$.

Proof. Assume tha the solutions converge. Substitute $\hat{\phi}_{j}=0, j=m^{\prime}+1$, $\cdots, m$ in (3.17). Then

$$
\sum_{k=j}^{m^{\prime}} p_{2, k} \cdot \hat{\phi}_{m^{\prime}+j-k} \equiv 0, \quad j=1, \cdots, m-m^{\prime} \text {. }
$$

When $j=m^{\prime} \leqq m-m^{\prime}$, we have $p_{2, m^{\prime}} \cdot \hat{\phi}_{m^{\prime}} \equiv 0$. Since $p_{2, m^{\prime}}$ is a polynomial with $p_{2, m^{\prime}} \neq 0$ and $\hat{\phi}_{m^{\prime}}$ is a continuous function, it implies that $\hat{\phi}_{m^{\prime}} \equiv 0$. Hence

$$
\sum_{k=j+1}^{m^{\prime}} p_{2, k} \cdot \hat{\phi}_{m^{\prime}+j-k} \equiv 0, j=1, \cdots, m-m^{\prime} .
$$

When $j=m^{\prime}-1$, we have $p_{2, m^{\prime}} \cdot \hat{\phi}_{m^{\prime}-1} \equiv 0$. Since $p_{2, m^{\prime}} \neq 0$, it implies that $\hat{\phi}_{m^{\prime}-1}$ $\equiv 0$. Repeating this process $m^{\prime}$ times, we come to the conclusion. The converse is trivial.

Q.E.D.

Corollary 3.6. Let the same assumptions as in Proposition be satisfied. Let $\phi_{j}=0, j=1, \cdots, l-1, l+1, \cdots, m$.

(Case 1). The case when $1 \leqq l \leqq m^{\prime}$. Assume that there exists a positive integer $j^{\prime}$ with $m^{\prime}+1-l \leqq j^{\prime} \leqq \min \left\{m-l, m^{\prime}\right\}$ such that $p_{2, j^{\prime}} \neq 0$. Then the solutions $u_{\varepsilon}(x ; \Phi)$ of $(1.1)$ converge in $C\left(\mathbb{R}^{n}\right)$ for this Cauchy data if and only if $\phi_{l}=0$.

(Case 2$)$. The case when $m^{\prime}+1 \leqq l \leqq m$. The solutions $u_{\varepsilon}(x ; \Phi)$ of $(1.1)$ converge in $C\left(\boldsymbol{R}^{n}\right)$ for this Cauchy data if and only if $\phi_{l}=0$.

Proof. Assume that the solutions converge. Let $1 \leqq l \leqq m^{\prime}$. Substitute $\hat{\phi}_{j}=0, j=1, \cdots, l-1, l-1, \cdots, m$ in (3.17). Then $p_{2, m^{\prime}+j-l} \cdot \hat{\phi}_{l} \equiv 0, j=1, \cdots, m-$ $m^{\prime}$. We use the case when $j=j^{\prime}+l-m^{\prime}$, that is, $p_{2, j^{\prime}} \cdot \hat{\phi}_{l} \equiv 0$. Since $p_{2, j^{\prime}} \neq 0$, it implies that $\hat{\phi_{l}} \equiv 0$. When $m^{\prime}+1 \leqq l \leqq m$, Corollary 3.4 implies the conclusion. The converse is trivial.

Q.E.D

\section{The Admissibility of the Cauchy Data}

In this section, we shall remove the dependency on $\varepsilon$ from (3.1) and 
classify the Cauchy data space $\mathrm{F}^{-1}\left(C_{0}^{\infty}\left(B_{0}\right)\right)^{m}$ into two classes in the two special cases mentioned in $\S 1$. One class is the set of all the Cauchy data $\Phi$ with which the solutions $u_{\mathrm{\varepsilon}}(x ; \Phi)$ of (1.1) converge in $C\left(\boldsymbol{R}^{n}\right)$ and the other is the complement.

Denote the characteristic roots of $\varepsilon^{n-m^{\prime}} \cdot P_{1}(\xi)+P_{2}(\xi)=0$ with respect to $\xi_{1}$ by $\tau_{j}\left(\varepsilon, \xi^{\prime}\right), j=1, \cdots, m$, those of $P_{1}(\xi)=0$ by $v_{j}\left(\xi^{\prime}\right), j=1, \cdots, m$, and those of $P_{2}(\xi)=0$ by $\sigma_{j}\left(\xi^{\prime}\right), j=1, \cdots, m^{\prime}$. Then we have the following lemma.

Lemma 4.1. Let $B$ be a fixed arbitrary open set in $\mathbb{R}^{n-1}$. For every integer $i$ with $1 \leqq i \leqq m^{\prime}$, there eixsts an integer $j$ with $1 \leqq j \leqq m$ such that $\sigma_{i} \equiv v_{j}$ in $B$ if and only if $P_{1}(\xi)$ is divided by $P_{2}(\xi)$ in the polynomial ring $\mathbb{C}[\xi]$.

Proof. We may assume that $\sigma_{j} \equiv v_{j}, j=1, \cdots, m^{\prime}$ in $B$. Applying Lemma 2.3 for $X_{i}=\sigma_{i}, i=1, \cdots, m^{\prime}$ and $Y_{j}=v_{j+m^{\prime}}, j=1, \cdots, m^{\prime \prime}$, we have in $B$ for $k=1, \cdots, m^{\prime \prime}$

$$
\begin{gathered}
S_{k}\left(v_{m^{\prime}+1}, \cdots, v_{m}\right)=S_{k}\left(\sigma_{1}, \cdots, \sigma_{m^{\prime}}, v_{m^{\prime}+1}, \cdots, v_{m}\right) \\
-\sum_{j=0}^{k-1} S_{k-j}\left(\sigma_{j}, \cdots, \sigma_{m^{\prime}}\right) \cdot S_{j}\left(v_{m^{\prime}+1}, \cdots, v_{m}\right) .
\end{gathered}
$$

Since

$$
S_{k}\left(\sigma_{1}, \cdots, \sigma_{m^{\prime}}, v_{m^{\prime}+1}, \cdots, v_{m}\right)=(-1)^{k} \cdot p_{1, k}\left(\xi^{\prime}\right) / p_{1,0}, \quad k=1, \cdots, m
$$

and

$$
S_{k}\left(\sigma_{1}, \cdots, \sigma_{m^{\prime}}\right)=(-1)^{k} \cdot p_{2, k}\left(\xi^{\prime}\right) / p_{2,0}, \quad k=1, \cdots, m^{\prime}
$$

are polynomials of $\xi^{\prime}$, we have inductively that $S_{k}\left(v_{n^{\prime}+1}, \cdots, v_{m}\right), k=1, \cdots, m^{\prime \prime}$ have polynomial representations in $B$. Hence

$$
\begin{aligned}
& P_{1}(\xi) / P_{2}(\xi)=\left(P_{1,0} / p_{2,0}\right) \cdot \prod_{k=1}^{m^{\prime \prime}}\left(\xi_{1}-v_{k+m^{\prime}}\right) \\
& \quad=\left(p_{1,0} / p_{2,0}\right) \cdot \sum_{k=0}^{m^{\prime \prime}}(-1)^{k} \cdot S_{k}\left(v_{m^{\prime}+1}, \cdots, v_{m}\right) \cdot \xi_{1}{ }^{m^{\prime \prime}-k}
\end{aligned}
$$

has a polynomial representation in $\mathbb{R} \times B$. Thus there exists a polynomial $P_{3}(\xi)$ such that $P_{1}(\xi) / P_{2}(\xi)=P_{3}(\xi)$ in $\mathbb{R} \times B$, which remains true in $\mathbb{R}^{n}$. The converse is trivial.

Q.E.D

Theorem A. Assume that Condition 1.2 is not satisfied. Let Assumption 1.1 be satisfied and $B_{0}$ be the open ball in Remark to Assumption 1.1. Let the Cauchy data $\Phi=\left(\phi_{1}, \cdots, \phi_{m}\right)$ of $(1.1)$ belong to $\mathrm{F}^{-1}\left(C_{0}^{\infty}\left(B_{0}\right)\right)^{m}$.

(Case 1). The case when for every integer $i$ with $1 \leqq i \leqq m^{\prime}$ there exists an integer $j$ with $1 \leqq j \leqq m$ such that $\sigma_{i} \equiv v_{j}$ in $B_{0}$, that is, when $P_{1}(\xi)$ is divided by $P_{2}(\xi)$ in the polynomial ring $\mathbb{C}[\xi]$. The solutions $u_{\varepsilon}(x ; \Phi)$ converge in $C\left(\mathbb{R}^{n}\right)$ if and 
only if the Cauchy data $\Phi$ satisfy (3.19). In this case, the solutions $u_{\mathrm{a}}(x ; \Phi)$ are identically equal to the solution $u_{0}\left(x ; \Phi^{\prime}\right)$ of $(2.1)$.

(Case 2). The case when $\sigma_{1} \neq v_{j}$, for all $j=1, \cdots, m$ and for every integer $i$ with $2 \leqq i \leqq m^{\prime}$ there exists an integer $j$ with $1 \leqq j \leqq m$ such that $\sigma_{i} \equiv v_{j}$ in $B_{0}$. The solutions $u_{\mathrm{z}}(x ; \Phi)$ converge in $C\left(\boldsymbol{R}^{n}\right)$ if and only if the Cauchy data $\Phi$ satisfy

$$
\sum_{k=0}^{m^{\prime}-1}(-1)^{k} \cdot S_{k}\left(\sigma_{2}, \cdots, \sigma_{m^{\prime}}\right) \cdot \hat{\phi}_{m^{\prime}+j-k}=0, j=0, \cdots, m-m^{\prime} .
$$

Here if $m^{\prime}=1$, then $S_{0}=1$ and (4.1) is equivalent to $\Phi=(0, \cdots, 0)$. In this case, the solutions $u_{\varepsilon}(x ; \Phi)$ depend on $\varepsilon$ in general.

In order to prove Theorem A, we need the following lemma.

Lemma 4.2. Assume that the characteristic roots satisfy $\tau_{j}\left(\varepsilon, \xi^{\prime}\right) \equiv \sigma_{j}\left(\xi^{\prime}\right)$, $j=1, \cdots, m^{\prime}$ in $B_{0}$. Then for the Cauchy data satisfying (3.17),

$$
\frac{D_{l}\left(\tau_{1}, \cdots, \tau_{m} ; \hat{\phi}_{1}, \cdots, \hat{\phi}_{m}\right)}{D_{0}\left(\tau_{1}, \cdots, \tau_{m}\right)}=\frac{D_{l}\left(\sigma_{1}, \cdots, \sigma_{m^{\prime}} ; \hat{\phi}_{1}, \cdots, \hat{\phi}_{m^{\prime}}\right)}{D_{0}\left(\sigma_{1}, \cdots, \sigma_{m^{\prime}}\right)},
$$

$l=1, \cdots, m^{\prime}$

Proof. Put for $l=1, \cdots, m^{\prime}$ and for $k=1, \cdots, m$

$$
d_{l, k}={ }^{t}\left(\tau_{1}{ }^{k-1}, \cdots, \tau_{l-1}{ }^{k-1}, \hat{\phi}_{k}, \tau_{l+1}{ }^{k-1}, \cdots, \tau_{m}{ }^{k-1}\right) .
$$

Since for $i=1, \cdots, m^{\prime}$ and $j=1, \cdots, m-m^{\prime}$

$$
\sum_{k=0}^{m^{\prime}} p_{2, k} \cdot \tau_{i}^{{ }^{\prime}+j-k-1}=\sigma_{i}{ }^{j-1} \cdot P_{2}\left(\sigma_{i}, \xi^{\prime}\right)=0,
$$

it implies that

$$
\sum_{k=0}^{n^{\prime}} p_{2, k} \cdot d_{l, m^{\prime}+j-k}={ }^{t}\left(0, \cdots, 0, \tau_{m m^{\prime}+1}^{j-1} \cdot P_{2}\left(\tau_{m^{\prime}+1}, \xi^{\prime}\right), \cdots, \tau_{m}^{j-1} \cdot P_{2}\left(\tau_{m}, \xi^{\prime}\right)\right) .
$$

Then

$$
\begin{aligned}
& D_{l}\left(\tau_{1}, \cdots, \tau_{m} ; \hat{\phi}_{1}, \cdots, \hat{\phi}_{m}\right)=\operatorname{det}^{t}\left(d_{l, k} ; k \rightarrow 1, \cdots, m\right) \\
& \quad=\operatorname{det}\left(d_{l, 1}, \cdots, d_{l, m-1}, p_{2,0}{ }^{-1} \cdot \sum_{k=0}^{m^{\prime}} p_{2, k} \cdot d_{l, m-k}\right) \\
& \quad=\operatorname{det}\left(d_{l, 1}, \cdots, d_{l, m^{\prime}}, p_{2,0}{ }^{-1} \cdot \sum_{k=0}^{m^{\prime}} p_{2, k} \cdot d_{l, m^{\prime}+1-k}, \cdots, p_{2,0}{ }^{-1} \cdot \sum_{k=0}^{m^{\prime}} p_{2, k} \cdot d_{l, m-k}\right) \\
& \quad=D_{l}\left(\sigma_{1}, \cdots, \sigma_{m} ; \hat{\phi}_{1}, \cdots, \hat{\phi}_{m^{\prime}}\right) \cdot D_{0}\left(\tau_{m^{\prime}+1}, \cdots, \tau_{m}\right) \cdot\left(\prod_{k=m^{\prime}+1}^{m} P_{2}\left(\tau_{k}, \xi^{\prime}\right)\right) \cdot p_{2,0}{ }^{-{ }^{\prime}}, m^{\prime}
\end{aligned}
$$

where we use the fact that $P_{2}\left(\tau_{k}, \xi^{\prime}\right) \neq 0$ in $B_{0}, k=m^{\prime}+1, \cdots, m$ for sufficiently small $\varepsilon$, because $\tau_{j}\left(\varepsilon, \xi^{\prime}\right), j=1, \cdots, m$ are distinct in $B_{0}$ for sufficiently small $\varepsilon$. By the same argument,

$$
\begin{aligned}
& D_{0}\left(\tau_{1}, \cdots, \tau_{m}\right) \\
& \quad=D_{0}\left(\sigma_{1}, \cdots, \sigma_{m^{\prime}}\right) \cdot D_{0}\left(\tau_{m^{\prime}+1}, \cdots, \tau_{m}\right) \cdot\left(\Pi_{k=m^{\prime}+1}^{m} P_{2}\left(\tau_{k}, \xi^{\prime}\right)\right) \cdot p_{2,0}{ }^{-m+m^{\prime}} .
\end{aligned}
$$


Since $D_{0}\left(\tau_{m^{\prime}+1}, \cdots, \tau_{m}\right) \cdot\left(\prod_{k=m^{\prime}+1}^{m} P_{2}\left(\tau_{k}, \xi^{\prime}\right)\right) \neq 0$ in $B_{0}$ for sufficiently small $\varepsilon$ except $\varepsilon=0$, we come to the concuusion.

Q.E.D.

\section{Proof of Theorem A.}

(Case 1). By Remark to Lemma 2.5, we have

$$
\tau_{j}\left(\varepsilon, \xi^{\prime}\right) \equiv \sigma_{j}\left(\xi^{\prime}\right), j=1, \cdots, m^{\prime} \text { in } B_{0} .
$$

Substitute $\sigma_{j}$ for $\tau_{j}, j=1, \cdots, m^{\prime}$ in (3.1) and (3.2). Then (3.1) are equivalent to (3.19) when (4.2) are satisfied. Lemma 4.2 and (3.2) imply that $u_{\mathrm{g}}(x ; \Phi)=$ $u_{0}\left(x ; \Phi^{\prime}\right)$.

(Case 2). By Remark to Lemma 2.5, we have $s_{1,2} \neq 0$ and

$$
\tau_{j}\left(\varepsilon, \xi^{\prime}\right) \equiv \sigma_{j}\left(\xi^{\prime}\right), \quad j=2, \cdots, m^{\prime} .
$$

Substitute $\sigma_{j}$ for $\tau_{j} j=2, \cdots, m^{\prime}$ in (3.1) and (3.2). Since

$$
S_{k}\left(\tau_{1}, \sigma_{2}, \cdots, \sigma_{m^{\prime}}\right)=S_{k}\left(\sigma_{2}, \cdots, \sigma_{m^{\prime}}\right)+\tau_{1} \cdot S_{k-1}\left(\sigma_{2}, \cdots, \sigma_{m^{\prime}}\right),
$$

it implies that (3.1) are equivalent to

$$
\begin{aligned}
& \sum_{k=0}^{m^{\prime}}(-1)^{k} \cdot S_{k}\left(\sigma_{2}, \cdots, \sigma_{m^{\prime}}\right) \cdot \hat{\phi}_{m^{\prime}+j-k} \\
& \quad+\tau_{1} \cdot \sum_{k=0}^{m^{\prime}}(-1)^{k} \cdot S_{k-1}\left(\sigma_{2}, \cdots, \sigma_{m^{\prime}}\right) \cdot \hat{\phi}_{m^{\prime}+j-k} \\
& \quad=\sum_{k=0}^{m^{\prime}-1}(-1)^{k} \cdot S_{k}\left(\sigma_{2}, \cdots, \sigma_{m^{\prime}}\right) \cdot\left(\hat{\phi}_{m^{\prime}+j-k}-\tau_{1} \cdot \hat{\phi}_{m^{\prime}+j-k-1}\right) \equiv 0,
\end{aligned}
$$

$j=1, \cdots, m-m^{\prime}$. Substitute (2.10) for $\tau_{1}$ in (4.4). Then the coefficients of $\varepsilon^{0}$ in (4.4) are

$$
\sum_{k=0}^{m^{\prime}-1}(-1)^{k} \cdot S_{k}\left(\sigma_{2}, \cdots, \sigma_{m^{\prime}}\right) \cdot\left(\hat{\phi}_{m^{\prime}+j-k}-\sigma_{1} \cdot \hat{\phi}_{m^{\prime}+j-k-1}\right)
$$

and those of $\varepsilon^{m-m^{\prime}}$ are

$$
-S_{1,2} \cdot \sum_{k=0}^{m^{\prime}-1}(-1)^{k} \cdot S_{k}\left(\sigma_{2}, \cdots, \sigma_{m^{\prime}}\right) \cdot \hat{\phi}_{m^{\prime}+j-k-1},
$$

which must be zero. Since $s_{1,2} \neq 0$ and $s_{1,2}$ is an analytic function in $B_{0}$, it implies that

$$
\sum_{k=0}^{m^{\prime}-1}(-1)^{k} \cdot S_{k}\left(\sigma_{2}, \cdots, \sigma_{m^{\prime}}\right) \cdot \hat{\phi}_{m^{\prime}+j-k-1}=0
$$

in $\left\{\xi^{\prime} \in B_{0} ; s_{1,2}\left(\xi^{\prime}\right) \neq 0\right\}$ and therefore in $B_{0}$. By substituting (4.6) in (4.5), we have (4.1), which are equivalent to (3.1). Obviously (3.2) depends on $\varepsilon$ in general. Thus we come to the conclusion.

Q.E.D.

Remark. When $P_{1}(\xi)$ is divided by $P_{2}(\xi)$ in $\mathbb{C}[\xi]$, there exists a datum $\Phi^{\prime \prime}$ for an arbitrary datum $\Phi^{\prime}$ such that the solutions converge. In fact, we may take $\phi_{j}=D_{1}^{j-1} u_{0}\left(x ; \Phi^{\prime}\right), j=m^{\prime}+1, \cdots, m$, which belong to $\mathrm{F}^{-1}\left(C_{0}^{\infty}\left(B_{0}\right)\right)$ and are 
independent of $\varepsilon$. Corollary 3.4 implies the uniqueness of such data $\Phi^{\prime \prime}$ as the solutions converge and the Cauchy-Kowalewski theorem implies the uniqueness of the solutions for fixed $\varepsilon$. Hence we can prove (Case 1) without Lemma 4.2. Our aim to use Lemma 4.2 is to prove (Case 1) only with algebraic calculations.

Theorem B. Let $m^{\prime}=1$ or $\left(m^{\prime}=2\right.$ and $\left.m \geqq 4\right)$. Assume that Condition 1.2 is not satisfied. Let Assumption 1.1 be satisfied and $B_{0}$ be the open ball in Remark to Assumption 1.1. Let the Cauchy data $\Phi=\left(\phi_{1}, \cdots, \phi_{m}\right)$ of $(1.1)$ belong to $\mathrm{F}^{-1}\left(C_{0}^{\infty}\left(B_{0}\right)\right)^{m}$.

(Case 1). The case when $m^{\prime}=1$.

(1-a) If there exists an integer $j$ with $1 \leqq j \leqq m$ such that $\sigma_{1} \equiv v_{j}$ in $B_{0}$, then the solutions $u_{\mathrm{\varepsilon}}(x ; \Phi)$ converge in $C\left(\boldsymbol{R}^{n}\right)$ if and only if the Cauchy data $\Phi$ satisfy

$$
\hat{\phi}_{j}\left(\xi^{\prime}\right)=\sigma_{i}{ }^{j-1}\left(\xi^{\prime}\right) \cdot \hat{\phi}_{1}\left(\xi^{\prime}\right), j=2, \cdots, m \text {. }
$$

(1-b) If $\sigma_{1} \neq v_{j}, j=1, \cdots, m$ in $B_{0}$. Then the solutions $u_{\varepsilon}(x ; \Phi)$ converge in $C\left(\boldsymbol{R}^{n}\right)$ if and only if $\Phi=(0, \cdots, 0)$.

(Case 2). The case when $m^{\prime}=2$ and $m \geqq 4$.

(2-a) If there exist integers $j_{1}$ and $j_{2}$ with $1 \leqq j_{1}, j_{2} \leqq m$ such that $\sigma_{1} \equiv v_{j_{1}}$ and $\sigma_{2} \equiv v_{j_{2}}$ in $B_{0}$ then the solutions $u_{\varepsilon}(x ; \Phi)$ converge in $C\left(\boldsymbol{R}^{n}\right)$ if and only if the Cauchy data $\Phi$ satisfy (3.19) for $m^{\prime}=2$.

(2-b) If there exists an integer $j$ with $1 \leqq j \leqq m$ such that $\sigma_{1} \equiv v_{j}$ and $\sigma_{2} \neq v_{k}$, for all $k=1, \cdots, m$ in $B_{0}$, then the solutions $u_{\mathrm{s}}(x ; \Phi)$ converge in $C\left(\boldsymbol{R}^{n}\right)$ if and only if the Cauchy data $\Phi$ satisfy (4.7).

(2-c) If $\sigma_{i} \neq v_{j}, i=1,2$ and $j=1, \cdots, m$ in $B_{0}$. Then the solutions $u_{\varepsilon}(x ; \Phi)$ converge in $C\left(\boldsymbol{R}^{n}\right)$ if and only if $\Phi=(0, \cdots, 0)$.

Except $(2-\mathrm{b})$, the solutions $u_{\varepsilon}(x ; \Phi)$ are identically equal to the solution $u_{0}\left(x ; \Phi^{\prime}\right)$ of $(1.2)$.

In order to prove Theorem $B$, we need the following lemma.

Lemma 4.3. Let the same assumptions as in Proposition be satisfied. Then the following three conditions are equivalent.

(1) There exist an analytic function $\gamma\left(\xi^{\prime}\right)$ in $B_{0}$ and a $C_{0}^{\infty}\left(B_{0}\right)$-function $\beta\left(\xi^{\prime}\right)$ with $\beta\left(\xi^{\prime}\right) \neq 0$ such that the solutions of (1.1) converge for the Cauchy data satisfying

$$
\hat{\phi}_{j}\left(\xi^{\prime}\right)=\beta\left(\xi^{\prime}\right) \cdot \gamma\left(\xi^{\prime}\right)^{j-1}, \quad j=1, \cdots, m
$$


This implies that $\hat{\phi}_{1}=\beta$.

(2) There exists integers $i$ with $1 \leqq i \leqq m^{\prime}$ and $j$ with $1 \leqq j \leqq m$ such that

$$
\sigma_{i}\left(\xi^{\prime}\right) \equiv v_{j}\left(\xi^{\prime}\right) \text { in } B_{0}
$$

Here $v_{k}, k=1, \cdots, m$ are characteristic roots of $P_{1}(\xi)=0$ with respect to $\xi_{1}$ and $\sigma_{k}, k=1, \cdots, m^{\prime}$ are those of $P_{2}(\xi)=0$.

(3) There exists an analytic function $\gamma\left(\xi^{\prime}\right)$ in $B_{0}$ such that for all the $C_{0}^{\infty}\left(B_{0}\right)$ functions $\beta\left(\xi^{\prime}\right)$, the solutions of (1.1) converge for the Cauchy data satisfying (4.8).

Proof. We shall show that $(1) \rightarrow(2) \rightarrow(3) \rightarrow(1)$.

First step: $(1) \rightarrow(2)$. Substitute (4.8) in (3.17). Then

$$
\sum_{k=0}^{m^{\prime}} p_{2, k}\left(\xi^{\prime}\right) \cdot \beta\left(\xi^{\prime}\right) \cdot \gamma\left(\xi^{\prime}\right)^{m^{\prime}-k} \equiv 0 \text {. }
$$

Since

$$
\sum_{k=0}^{m^{\prime}} p_{2, k}\left(\xi^{\prime}\right) \cdot \gamma\left(\xi^{\prime}\right)^{m^{\prime}-k}=p_{2,0} \cdot \prod_{k=1}^{m^{\prime}}\left(\gamma\left(\xi^{\prime}\right)-\sigma_{k}\left(\xi^{\prime}\right)\right),
$$

it implies that if $\beta\left(\xi_{1}^{\prime}\right) \neq 0$, then there exists a neighbourhood $B_{1}$ of $\xi_{1}^{\prime}$ such that $\Pi_{k=1}^{m^{\prime}}\left(\gamma\left(\xi^{\prime}\right)-\sigma_{k}\left(\xi^{\prime}\right)\right) \equiv 0$ in $B_{1}$. Hence there exists an integer $i$ such that $r\left(\xi^{\prime}\right) \equiv$ $\sigma_{i}\left(\xi^{\prime}\right)$ in $B_{1}$. The analyticity implies that $r\left(\xi^{\prime}\right) \equiv \sigma_{i}\left(\xi^{\prime}\right)$ in $B_{0}$. We may assume that $i=1$. We have for $j=1, \cdots, m^{\prime}-1$,

$$
S_{j}\left(\tau_{1}, \cdots, \tau_{m^{\prime}}\right)=S_{j}\left(\tau_{2}, \cdots, \tau_{m^{\prime}}\right)+\tau_{1} \cdot S_{j-1}\left(\tau_{2}, \cdots, \tau_{m^{\prime}}\right) .
$$

Then (3.1) for $j=1$ implies that

$$
\begin{aligned}
& \sum_{j=0}^{m^{\prime}}(-1)^{j} \cdot S_{j}\left(\tau_{2}, \cdots, \tau_{m^{\prime}}\right) \cdot \hat{\phi}_{m^{\prime}+1-j} \\
& \quad+\tau_{1} \cdot \sum_{j=0}^{m^{\prime}}(-1)^{j} \cdot S_{j-1}\left(\tau_{2}, \cdots, \tau_{m^{\prime}}\right) \cdot \hat{\phi}_{m^{\prime}+1-j} \\
& \quad=\sum_{j=0}^{m^{\prime}-1}(-1)^{j} \cdot S_{j}\left(\tau_{2}, \cdots, \tau_{m^{\prime}}\right) \cdot\left(\hat{\phi}_{m^{\prime}+1-j}-\tau_{1} \cdot \hat{\phi}_{m^{\prime}-j}\right) \equiv 0 .
\end{aligned}
$$

Since

$$
\hat{\phi}_{m^{\prime}+1-j}-\tau_{1} \cdot \hat{\phi}_{m^{\prime}-j}=-S_{1,2} \cdot \beta \cdot \sigma_{1}^{m^{\prime}-1-j} \cdot \varepsilon^{m-m^{\prime}}+O\left(\varepsilon^{2 m-2 m^{\prime}}\right),
$$

(4.11) implies that

$$
-S_{1,2} \cdot \varepsilon^{m-m^{\prime}} \cdot \beta \cdot \sum_{j=0}^{m^{\prime}-1}(-1)^{j} \cdot S_{j}\left(\tau_{2}, \cdots, \tau_{m^{\prime}}\right) \cdot \sigma_{1}^{m^{\prime}-1-j}+O\left(\varepsilon^{2 m-2 m^{\prime}}\right) \equiv 0 .
$$

The least order of $\varepsilon$ is $m-m^{\prime}$ and the coefficient of $\varepsilon^{m-m^{\prime}}$ is

$$
-S_{1,2} \cdot \beta \cdot \sum_{j=0}^{m^{\prime}-1}(-1)^{j} \cdot S_{j}\left(\sigma_{2}, \cdots, \sigma_{m}\right) \cdot \sigma_{1}{ }^{m^{\prime}-1-j},
$$

which must be zero. Since $\beta\left(\xi^{\prime}\right) \neq 0$ and 


$$
\sum_{j=0}^{m^{\prime}-1}(-1)^{j} \cdot S_{j}\left(\sigma_{2}, \cdots, \sigma_{m^{\prime}}\right) \cdot \sigma_{1}^{m^{\prime}-1-j}=\prod_{j=2}^{m^{\prime}}\left(\sigma_{1}-\sigma_{j}\right) \neq 0 \text { in } B_{0},
$$

the analyticity of $s_{1,2}$ in $B_{0}$ implies that $s_{1,2}\left(\xi^{\prime}\right) \equiv 0$ in $B_{0}$. Then $P_{1}\left(\sigma_{1}, \xi^{\prime}\right) \equiv 0$ in $B_{0}$, which is equivalent to (4.9) for $i=1$.

Second step: $(2) \rightarrow(3)$. If (4.9) is satisfied for $i=1$, then we may assume that $\tau_{1}\left(\varepsilon, \xi^{\prime}\right) \equiv \sigma_{1}\left(\xi^{\prime}\right) \equiv \gamma\left(\xi^{\prime}\right)$ in $B_{0}$ and that $\hat{\Phi}=\left(\hat{\phi}_{1}, \cdots, \hat{\phi}_{m}\right)=\hat{\phi}_{1} \cdot\left(1, \tau_{1}, \cdots \tau_{1}{ }^{m-1}\right)$, where $\hat{\phi}_{1}$ is an arbitrary $C_{0}^{\infty}\left(B_{0}\right)$-function. Hence for $l=2, \cdots, m$

$$
D_{l}\left(\tau_{1}, \cdots, \tau_{m} ; \hat{\phi}_{1}, \cdots, \hat{\phi}_{m}\right)=\hat{\phi}_{1} \cdot D_{0}\left(\tau_{1}, \cdots, \tau_{l-1}, \tau_{1}, \tau_{l+1}, \cdots, \tau_{m}\right) \equiv 0,
$$

and

$$
D_{1}\left(\tau_{1}, \cdots, \tau_{m} ; \hat{\phi}_{1}, \cdots, \hat{\phi}_{m}\right)=\hat{\phi}_{1} \cdot D_{0}\left(\tau_{1}, \cdots, \tau_{m}\right)
$$

Thus

$$
u_{\varepsilon}(x ; \Phi)=\mathrm{F}^{-1}\left(\phi_{1}\left(\xi^{\prime}\right) \cdot \exp i \sigma_{1}\left(\xi^{\prime}\right) x_{1}\right),
$$

which is the solution of (1.2). Since the solutions $u_{\varepsilon}$ are independent of $\varepsilon$, the solutions $u_{\varepsilon}$ converge.

Third step: (3) $\rightarrow(1)$. This is trivial.

Q.E.D.

Proof of Theorem B. (Case 1). First we assume that the solutions $u_{\varepsilon}$ converge. In this case, (3.18) implies (4.7). When $\hat{\phi}_{1}\left(\xi^{\prime}\right) \equiv 0,(4.7)$ implies that $\Phi=(0, \cdots, 0)$ and the solutions $u_{\varepsilon}$ are identically zero. When $\hat{\phi}_{1}\left(\xi^{\prime}\right) \neq 0$, Lemma 4.3 implies that there exists an integer $j$ with $1 \leqq j \leqq m$ such that $\sigma_{1} \equiv v_{j}$ in $B_{0}$. Hence if there exist data $\Phi$ such that $\hat{\phi}_{1}\left(\xi^{\prime}\right) \neq 0$ and the solutions converge, then it leads to $(1-a)$. Otherwise it leads to $(1-b)$. The converse can be proved with the same argument as in Lemma 4.3.

(Case 2). The case (2-a) is the special case of (Case 1) in Theorem A. The case (2-b) is that of (Case 2) in Theorem A, where we must exchange $\sigma_{1}$ for $\sigma_{2}$. Therefore we may assume that $s_{1,2} \neq 0$ and $s_{2,2} \neq 0$ in $B_{0}$. Put $s=$ ${ }^{t}\left(s_{1,2}, s_{2,2}\right)$ and $A_{1}=\left(\hat{\phi}_{j+2-k} ; j \downarrow 1,2, k \rightarrow 1,2\right)$. Assume that the solutions converge. Then Corollary 3.3 implies that $A_{1} B s=0$. Since det $B \neq 0$ and $s \neq 0$ in $B_{0}$, it implies that det $A_{1}=0$, that is,

$$
\hat{\phi}_{2}^{2}=\hat{\phi}_{1} \cdot \hat{\phi}_{3} \text {. }
$$

By Corollary 3.2, we have

$$
p_{2,0} \cdot \hat{\phi}_{3}+p_{2,1} \cdot \hat{\phi}_{2}+p_{2,2} \cdot \hat{\phi}_{1}=0
$$

Then (4.12) and (4.13) implies that 


$$
p_{2,0} \cdot \hat{\phi}_{2}{ }^{2}+p_{2,1} \cdot \hat{\phi}_{11} \cdot \hat{\phi}_{2}+p_{2,2} \cdot \phi_{1}{ }^{2}=0 \text {. }
$$

Assume that $\phi_{1} \neq 0$. Divide (4.14) by $\phi_{1}$ and put $z=\hat{\phi}_{2} / \hat{\phi}_{1}$. Then

$$
p_{2,0^{\circ}} z^{2}+p_{2,1} \cdot z+p_{2,2}=P_{2}\left(z, \xi^{\prime}\right)=0 \text {. }
$$

Hence there exists an open ball $B_{1}$ with centre $\xi_{1}^{\prime}$ included in $B_{0}$ such that $\hat{\phi}_{1}\left(\xi^{\prime}\right)$ $\neq 0$ in $B_{1}$ and $z \equiv \sigma_{1}\left(\xi^{\prime}\right)$ or $z \equiv \sigma_{2}\left(\xi^{\prime}\right)$ in $B_{1}$. We may assume that $z \equiv \sigma_{1}\left(\xi^{\prime}\right)$, that is, $\hat{\phi}_{2}=\sigma_{1}{ }^{\circ} \hat{\phi}_{1}$ in $B_{1}$. Since (3.17) are equivalent to

$$
\begin{aligned}
& \hat{\phi}_{j+2}-\left(\sigma_{1}+\sigma_{2}\right) \cdot \hat{\phi}_{j+1}+\sigma_{1} \cdot \sigma_{2} \cdot \hat{\phi}_{j} \\
& \quad=\hat{\phi}_{j+2}-\sigma_{1} \cdot \hat{\phi}_{j+1}-\sigma_{2} \cdot\left(\hat{\phi}_{j+1}-\sigma_{1} \cdot \hat{\phi}_{j}\right)=0, j=1, \cdots, m-2,
\end{aligned}
$$

it implies that

$$
\hat{\phi}_{j+2}-\sigma_{1}{ }^{\circ} \hat{\phi}_{j+1}=0, \quad j=0, \cdots, m-2 \text { in } B_{1} .
$$

Choose $\beta_{1}$ in $C_{0}^{\infty}\left(B_{1}\right)$ satisfying $\beta_{1}\left(\xi_{1}^{\prime}\right) \neq 0$ and put $\beta=\beta_{1} \cdot \hat{\phi}_{1}$ and $\gamma=\sigma_{1}$. Then Remark to Proposition implies that the solutions converge for the Cauchy datum $\Phi_{1}$ satisfying

$$
\hat{\Phi}_{1}=\beta_{1} \cdot \hat{\phi}_{1} \cdot\left(1, \sigma_{1}, \cdots, \sigma_{1}{ }^{n-1}\right) .
$$

By applying Lemma 4.3 , we have $\sigma_{1}\left(\xi^{\prime}\right) \equiv \tau_{1}\left(\varepsilon, \xi^{\prime}\right) \equiv 0$ in $B_{0}$, which contradicts that $s_{1,2} \neq 0$. Hence $\hat{\phi}_{1} \equiv 0$. Then (4.12) and (4.13) implies that $\hat{\phi}_{2} \equiv \hat{\phi}_{3} \equiv 0$. Thus (4.16) implies that $\Phi=(0, \cdots, 0)$.

Q.E.D.

\section{The Weak Admissibility}

In this section, we shall prove Main Theorem.

Proof of Main Theorem. Assume that for every $\Phi^{\prime}$ in $\mathcal{A}^{\prime}$ there exists $\Phi^{\prime \prime}$ in $\mathcal{A}^{\prime \prime}$ such that the solutions $u_{\varepsilon}(x ; \Phi)$ of $(1.1)$ converge in $C\left(\mathbb{R}^{n}\right)$. We have only to show that it leads to (Case 1) in Theorem A. Assume that the condition in (Case 1) in Theorem $\mathrm{A}$ is not satisfied. We may assume that $s=$ $\left(s_{i, 2} ; i \downarrow 1, \cdots, m^{\prime}\right) \neq 0$. We can apply Corollary 3.3. Since $\operatorname{det} B \neq 0$, it implies that $B s \neq 0$. By (3.21) for $j=1$, we have $\left(\hat{\phi}_{m^{\prime}}, \cdots, \hat{\phi}_{1}\right) \cdot B s \equiv 0$. Hence $\Phi^{\prime}$ can not be arbitrary, which contradicts the $C$-weak admissibility. The converse is proved by (Case 1) in Theorem A.

Q.E.D.

Obvoously the $C$-admissibility implies the $C$-weak admissibility. When the Cauchy problems (1.1) are not $C$-weakly admissible, (1.1) are not suitable as a singular perturbation. Because the Cauchy data $\Phi^{\prime}$ of the reduced pro- 
blem (1.2) are restricted. One of the reasons why (1.2) does not admit adequate Cauchy data $\Phi^{\prime}$ is that the Cauchy data $\Phi^{\prime \prime}$ are independent of $\varepsilon$. If we allow that the Cauchy data $\Phi^{\prime \prime}$ change as $\varepsilon$, then we can find a sequence of solutions of (1.1) whose limit is the solution of (1.2). In fact, we may take the solutions satisfying

$$
\hat{u}_{\varepsilon}\left(x_{1}, \xi^{\prime}\right)=\sum_{j=0}^{m^{\prime}} \frac{D_{j}\left(\tau_{1}, \cdots, \tau_{m^{\prime}} ; \hat{\phi}_{1}, \cdots, \hat{\phi}_{m^{\prime}}\right)}{D_{0}\left(\tau_{1}, \cdots, \tau_{m^{\prime}}\right)} \cdot \exp i \tau_{j}\left(\varepsilon, \xi^{\prime}\right) x_{1} .
$$

When the Cauchy problems (1.1) are not $C$-admissible but $C$-weakly admissible, (1.1) are trivial as a singular perturbation. Because the solutions $u_{\varepsilon}(x ; \Phi)$ of (1.1) are identically equal to the solution $u_{0}\left(x ; \Phi^{\prime}\right)$ of (1.2). Thus the interesting cases are limited to the cases satisfying Condition 1.2.

When $\mathcal{A}^{\prime}$ is wider, there is a difficulty in the choise of $\mathcal{A}^{\prime \prime}$. But the proof of Corollary 3.2 suggests that $\Phi^{\prime \prime}$ is uniquely determined by $\Phi^{\prime}$ for wider data. There is another difficulty. For example, when $\hat{\Phi}$ belongs to $\mathcal{E}^{\prime}\left(B_{0}\right)^{m}$, the situation is so delicate that we can not analyze with algebraic methods only. In fact, we used the fact that the product of an analytic function not identically zero and a continuous function can not be identically zero except that the continuous function is identically zero.

\section{References}

[1] R. Ashino, The reducibility of the boundary conditions in the one-parameter family of elliptic linear boundary value problems I, Osaka J. Math,. 25 (1988), 737-757.

[2] - The reducibility of the boundary conditions in the one-parameter family of elliptic linear boundary value problems II, Osaka J. Math., 26 (1989), 535-556.

[3] , On the admissibility of singular perturbations in Cauchy problems, Osaka J. Math., 26 (1989), 387-398.,

[4] H. Weyl, The Classical Groups, their invariants and representations, second edition, Princceton, 1946. 
